Philosophie ANTIQUE

\section{Philosophie antique}

Problèmes, Renaissances, Usages

17 | 2017

Platon et la politique

\title{
Éphrem, Bardesane et Albinus sur les incorporels
}

Une confrontation entre le platonisme et le stoïcisme en milieu syriaque

\section{Izabela Jurasz}

\section{OpenEdition}

\section{Journals}

Édition électronique

URL : https://journals.openedition.org/philosant/292

DOI : 10.4000/philosant.292

ISSN : 2648-2789

Éditeur

Éditions Vrin

\section{Édition imprimée}

Date de publication : 1 novembre 2017

Pagination : 169-204

ISBN : 978-2-7574-1807-9

ISSN : $1634-4561$

\section{Référence électronique}

Izabela Jurasz, «Éphrem, Bardesane et Albinus sur les incorporels », Philosophie antique [En ligne], 17 | 2017, mis en ligne le 01 novembre 2018, consulté le 03 décembre 2022. URL : http:// journals.openedition.org/philosant/292 ; DOI : https://doi.org/10.4000/philosant.292

\section{(c) (i) (9)}

Creative Commons - Attribution - Pas d'Utilisation Commerciale - Pas de Modification 4.0 International - CC BY-NC-ND 4.0

https://creativecommons.org/licenses/by-nc-nd/4.0/ 


\section{ÉPHREM, BARDESANE ET ALBINUS SUR LES INCORPORELS Une confrontation entre le platonisme et le stoïcisme en milieu syriaque Izabela JuRASZ \\ Université Paris - Sorbonne, Centre Léon Robin}

RÉSUMÉ. Le Discours contre le Discours «De Domnus » rend compte d'un triple débat, dans lequel trois personnages interviennent sur la question des « incorporels », point difficile de la doctrine stoïcienne. La polémique rédigée par le stoïcien Bardesane, en réaction aux positions du platonicien Albinus, a été examinée par Éphrem le Syrien, chrétien et polémiste antibardesanite. Il est l'auteur du Discours et notre seule source d'information sur cette controverse. Or, la manière dont Éphrem aborde la question litigieuse est spontanément platonicienne, parfois proche du stoïcisme, mais fondamentalement, il s'agit d'une étonnante lecture théologique d'un thème qui fait débat entre les écoles philosophiques.

Summary. The Discourse against the Discourse 'De Domnus' is a three-part debate, in which three theologians discuss the question of «incorporeals », which is a difficultpoint of the Stoic doctrine. The polemic with the views of the Platonist Albinus, written by the Stoic Bardesan, was examined by Ephrem the Syrian, a Christian and an anti-Bardesanite polemicist, who is the author of the Discourse and our only source of information about this controversy. Ephrem addresses the contentious issue in a spontaneously Platonic manner, sometimes close to Stoicism, but fundamentally his Discourse is an astonishing theological reading of a theme that stirred debate between the two philosophical schools.

Philosophie antique, $\mathrm{n}^{\circ} 17$ (2017), 169-204 

Sachant le peu de considération qu'Éphrem le Syrien (†373) a eu pour la philosophie grecque*, on est surpris de lire son Discours contre le discours nommé « De Domnus » composépar Bardesane contre les platoniciens - même si ce texte figure au milieu d'un recueil hérésiologique connu sous le titre donné par l'éditeur anglais Prose Refutations against Mani, Marcion and Bardaisan ${ }^{1}$. Le Discours a réussi à attirer l'attention des chercheurs par la mention d'un écrit Sur les incorporels attribué au philosophe platonicien du II ${ }^{\mathrm{e}}$ siècle, Albinus (†190 ca. $)^{2}$. Dans le domaine des études syriaques, l'intérêt du Discours serait double, parce qu'il fournit un complément d'information sur la doctrine du philosophe chrétien Bardesane (†222 ca.) $)^{3}$ et aussi sur les écrits polémiques d'Éphrem, rédigés contre les différentes doctrines gnostiques $^{4}$. Il n'en demeure pas moins vrai que le Discours est rarement étudié,

${ }^{*}$ Je remercie Th. Bénatouïl et J.-B. Gourinat pour leurs remarques et leurs suggestions. Les erreurs sont miennes.

1. Le recueil comporte douze écrits dirigés contre Marcion ( $† 160$ ca.), Bardesane ( $† 222)$ et Mani (†276/277) ; voir vol. 1 Mitchell (éd.) 1912, vol. 2 Bevan \& Burkitt (éd.) 1921. Le Discours contre le « De Domnus » se trouve dans le vol. 2, p. 1-49 (texte), p. i-xxii (traduction anglaise). Le sens du titre «Domnus » (syriaque $: d$-dmnus) n'est pas clair. L'éditeur l'a pris comme nom d'un personnage, introduit par un dalet, par analogie avec les autres discours dédiés à un certain Hypatios : voir le commentaire de l'éditeur, vol. 2, p. cxii-cxiii.

2. L'écrit Sur les incorporels est mentionné uniquement dans le Discours éphremien. Voir Whittaker 1989a, p. 96-97; Gioè (éd.) 2002, p. 90-93.

3. Bardesane $(\dagger 222)$ est un philosophe chrétien d'origine syriaque, converti au christianisme au cours de ses études philosophiques. Les auteurs anciens lui attribuaient déjà des affinités d'une part avec la gnose valentinienne, d'autre part avec la philosophie stoïcienne. Voir Furlani 1937. Voir aussi : Hugonnard-Roche 1989; Teixidor 1994. Pour une vue générale : Teixidor 1992. La traduction allemande des parties lisibles du Discours contre le «De Domnus » est faite par Beck 1976. Les mentions du Discours dans les études sur Bardesane sont peu nombreuses. Dans sa monographie de Bardesane, H. J. W. Drijvers prétend qu'Éphrem mène la polémique antiplatonicienne - voir Drijvers 1966 p. 162-165. Voir aussi Camplani 1998 ; Possekel 2007.

4. Dans les études éphremiennes, le Discours est mentionné encore plus rarement ; voir Possekel 1999, p. 155-185. L'essentiel de la polémique antibardesanite d'Éphrem est exprimé 
sans doute en raison de son très mauvais état de conservation - le seul manuscrit existant, B.M. Add. 14623, est un palimpseste, illisible à quarante pour cent environ.

Pourquoi alors étudier la question des incorporels, à partir d'un texte aussi difficile d'accès ? Tout d'abord, parce que la doctrine des incorporels semble constituer le thème principal du Discours contre le «De Domnus ». Selon Éphrem lui-même, la raison directe qui l'a poussé à rédiger ces dizaines de pages est son désir de combattre les idées contenues dans un discours de Bardesane, intitulé De Domnus, texte polémique contre un écrit perdu d'Albinus Sur les incorporels. La critique éphremienne commence donc par la question des incorporels. De ce fait, Éphrem est notre seule source d'information sur le débat entre Albinus et Bardesane. S'il n'y a rien de particulièrement étrange dans le fait qu'un platonicien et un stoïcien échangent leurs avis au sujet des incorporels, il est, en revanche, extrêmement surprenant qu'un théologien chrétien, de surcroît peu féru de philosophie profane, veuille prendre part à ce débat et qu'il l'examine avec tant d'acharnement. La question des incorporels aurait-elle une portée théologique ? La réponse est difficile, parce que le Discours contre le «De Domnus » n'est pas rédigé dans une perspective théologique et c'est seulement dans sa conclusion que l'on découvre les critiques des positions de Bardesane sur Dieu. Il nous semble plutôt que, pour attirer l'attention d'Éphrem, l'échange entre Albinus et Bardesane devait se situer sur le terrain théologique au sens large du terme. Nous proposons cette hypothèse, malgré le caractère inévitablement fragmentaire des informations disponibles sur le déroulement du débat. Un indice supplémentaire vient du fait qu'Éphrem ne défend pas sa propre conception des incorporels - il semble même avoir une idée très floue de ce qu'est un incorporel stoïcien. Lorsqu'il examine les opinions de Bardesane et d'Albinus, c'est pour en vérifier la compatibilité avec la théologie chrétienne du $\mathrm{IV}^{\mathrm{e}}$ siècle. Cela explique sa manière confuse et approximative de se référer au platonisme et au stoïcisme. Toujours est-il que son analyse ne manque pas de rigueur, ni d'une certaine ingéniosité.

$\mathrm{Du}$ fait de nombreuses lacunes, le plan du Discours est presque impossible à reconstruire. Nous allons donc examiner les fragments relatifs aux incorporels dans l'ordre de leur apparition dans le texte, en essayant toutefois de les grouper par thèmes, dans le but d'éclairer les enjeux à la fois philosophiques et théologiques de cette polémique déployée à plusieurs niveaux. À chaque étape, nous montrerons aussi l'intérêt du fragment pour la connaissance de la diffusion des doctrines philosophiques grecques dans le milieu syriaque à l'époque qui va de Bardesane à Éphrem. 


\title{
1. Introduction au débat : Éphrem, Bardesane, Albinus, les platoniciens et les stö̈ciens (fr. 1-3)
}

\begin{abstract}
Fr. $1(6,41-7,20)$; Beck, fr. IV $(6,41-7,20)$
Tu sais alors qu' il est dit dans l'écrit De Domnus, que « les platoniciens $(d-b \bar{e} t$ Plāton) disent qu'il y a des somata $(\sigma \omega ́ \mu \alpha \tau \alpha)$ et des asomata $(\dot{\alpha} \sigma \omega ́ \mu \alpha \tau \alpha) \gg$; à savoir des corporels (gušmèe) et des incorporels (lā gšom). Ces arguments n'appartiennent pas aux platoniciens, bien qu' ils figurent dans les livres des platoniciens. Ces arguments sont stö̈ciens (stoyqē). Ils ont été exposés par Albinus, dans son livre intitulé Sur les incorporels ('l lā gušmēe), selon l'usage des sages et des philosophes qui, dans leurs écrits, exposaient d'abord les arguments auxquels ils adhéraient et ensuite se lançaient dans leurs débats pour réfuter les arguments des gens opposés à leurs affirmations.
\end{abstract}

Fr. $2(7,21-45)$; Beck, fr. V (7, 21-28)

C'est donc dans les écrits stö̈ciens et platoniciens (stoyqē w-platọn(i)qē), que « les platoniciens disent qu'il y a des somata ( $\sigma \omega ́ \mu \alpha \tau \alpha)$ et des asomata $(\dot{\alpha} \sigma \omega \dot{\omega} \alpha \tau \alpha) \gg$, et les stoïciens aussi < disent> la même chose. Ils ne sont pas d'accord sur le sens ( re $^{\prime}$ yannā), comme ils le sont sur les mots. Ainsi les platoniciens disent que les corporels et les incorporels existent en nature (b-kyanā) et en substance $(b-q n \bar{m} m \bar{a})$. Les stoïciens alors disent que tout ce qui existe en nature et en substance est corps (gušm $\bar{a})$, mais ils appellent incorporels tout ce qui n'existe pas en nature, mais qui peut être conçu dans l'intellect (re' yānāa).

Fr. 3 (7, 45-8, 14); Beck, fr. VI $(7,45-8,14)$

Voici, le philosophe des Syriens [i.e. Bardesane] s'est ridiculisé parmi les Syriens et les Grecs, non seulement parce qu'il était incapable de parler, mais aussi de montrer sa connaissance de l'enseignement de Platon. Par naïveté, il s'est hâté de blâmer Platon à propos des arguments des autres, alors

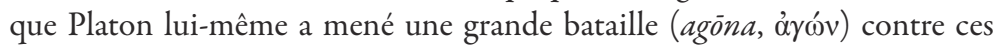
arguments que Bardesane croit appartenir à Platon.

Suivant la proposition d'E. Beck, nous divisons ce long passage en trois fragments, en raison des citations de l'écrit de Bardesane qui s'y trouvent. Quelques mots d'explication sur la manière éphremienne de citer et de commenter sont nécessaires. Le fragment commence par une phrase provenant de l'écrit de Bardesane, signalée par un terme lam, intraduisible marqueur de citation en syriaque. Sa présence ici veut dire qu'Éphrem cite l'écrit de Bardesane et qu'il cible la ou les phrases qui font l'objet de ses critiques; malheureusement, lam ne permet pas de déterminer avec précision l'étendue de la citation ${ }^{5}$. La citation est suivie d'une glose qui donne

5. On s'accorde sur le fait de limiter cette citation à l'affirmation suivante : « les platoniciens disent qu'il y a des somata et des asomata ». Il s'agit de la proposition faite par Bevan et Burkitt, reprise par E. Beck et U. Possekel. Voir Bevan \& Burkitt (éd.) 1921 p. iii (traduction); Beck 1976 p. 29 ; Possekel 1999 p. 156. Sur l'utilisation des citations signalées 
les termes syriaques correspondant aux $\sigma \omega ́ \mu \alpha \tau \alpha$ et $\alpha \sigma \omega ́ \mu \alpha \tau \alpha$ translittérés. En fait, l'utilisation des termes techniques grecs translittérés en syriaque est très habituelle à Bardesane ; c'est donc lui qui écrit somata et asomata ${ }^{6}$. Éphrem, en revanche, utilise les termes syriaques, qui sont les substantifs gušmā (pl. gušmè ) et là gušmā ou les adjectifs gšom et lā gšom. Il fait la même chose dans le fragment 2 , où les translittérations apparaissent dans la citation et les termes syriaques dans le commentaire qui la suit. Sur une vingtaine de passages concernant les incorporels, il n'y en a que cinq qui comportent des termes grecs transposés en caractères syriaques (somata, asomata, chrôma, smìyon, yukōna, parșōpa, sül(l)abia).

Le fragment 1 a fortement attiré l'attention car il contient l'information à propos du traité perdu d'Albinus ${ }^{7}$. Cette information est exceptionnelle pour Éphrem qui connaît très peu la philosophie grecque. En fait, à l'exception de Platon, il ne mentionne aucun philosophe par son nom - il parle de « platoniciens », de « stoïciens » ou de « philosophes » - et il ne cite aucun ouvrage, pas même par son titre. Qu'il parle d'Albinus et donne le titre de son écrit représente un cas unique, d’autant plus étonnant que ni le nom, ni les arguments d'Albinus ne sont évoqués dans le Discours. Pourtant, l'information sur cette œuvre est très précise : Albinus a exposé ses théories si on traduit littéralement : « dans le livre qui est appelé Sur les incorporels » (b-ktābeh hu d-metqrab 'llā gušmēè). Or, le titre de l'écrit d'Albinus est donné en syriaque (' l là gušmè) et il n'est pas une transcription de l'expression grecque. Si Éphrem recopie minutieusement les mots asomata dans les citations de Bardesane, pourquoi n'a-t-il pas fait la même chose pour un titre grec peu compliqué, comme $\pi \varepsilon \rho i ́ ~ \alpha ̊ \sigma \omega \mu \alpha ́ \tau \omega v$ ? Étant donné qu'Éphrem ne transcrit pas asomata en parlant de l'ouvrage d'Albinus, peut-être ce terme ne figurait-il pas dans son titre. Mais dans le même temps, l'analyse du vocabulaire concernant les êtres nommés lā gušmē montre que cette dénomination est très large, et qu'Éphrem mélange les $\alpha \sigma \omega ́ \mu \alpha \tau \alpha$ stoïciens avec les von $\tau \alpha$ platoniciens ${ }^{8}$.

par lam dans les Stances contre Bardesane (74, 80, 81, 82, 83), voir l'autre pièce du même recueil de Bevan \& Burkitt (éd.) 1921 p. 143-169 et lxvi-lxxix ; voir aussi Jurasz 2011-2012.

6. L'usage de termes techniques grecs par Bardesane se manifeste aussi dans le Livre des lois de pays. Un des termes clés de la doctrine bardesanite est èstuksē (transposition de $\sigma \tau o \chi \chi \varepsilon i \alpha)$, traduit systématiquement par Éphrem en syriaque par ityē. En outre, on y trouve

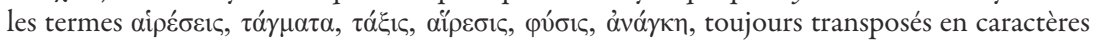
syriaques. Dans les fragments bardesanites du recueil de Prose Refutations - voir Mitchell (éd.) 1912 et Bevan \& Burkitt (éd.) 1921- on trouve d'autres exemples; dans le Discours :

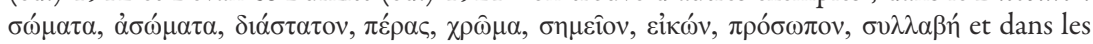

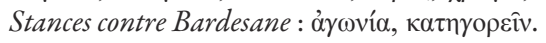

7. Le seul écrit conservé d'Albinus est le Prologue (Eisagoge) des dialogues de Platon; voir Le Corre 1956. Voir aussi Reis 1999.

8. Un cas semblable de cette attitude se trouve chez Sextus Empiricus ; voir Bréhier 1955. La comparaison avec Sextus est d'autant plus importante qu'E. Beck signale des 
Si l'information sur les platoniciens qui distinguent entre somata et asomata est une citation tirée du texte de Bardesane, on ne peut pas dire la même chose des renseignements sur l'origine stö̈cienne de la doctrine des incorporels, ainsi que de la brève caractérisation du traité d'Albinus. Sans aucun doute, Éphrem doit l'essentiel de sa connaissance du stoïcisme à Bardesane ; mais comment faut-il comprendre l'information sur l'ordonnancement de l'écrit d'Albinus, à savoir qu'il aurait exposé d'abord sa propre doctrine pour ensuite réfuter celle de ses adversaires stoïciens ?

L'exactitude de cette information a été discutée lorsque É. Orth a proposé d'attribuer à Albinus l'écrit Que les qualités sont incoporelles du Pseudo-Galien ; en réponse, M. Giusta a montré que ni le plan, ni le contenu de cet écrit ne correspondent aux questions débattues entre Éphrem et Bardesane9. É. Bréhier observe que de nombreuses informations sur la doctrine des incorporels proviennent de ceux-là mêmes qui l'ont contredite - des platoniciens (Plutarque), des académiciens et des sceptiques (Cicéron et Sextus Empiricus), des commentateurs d'Aristote (Ammonius, Alexandre d'Aphrodise, Simplicius $)^{10}$. Cependant, la remarque d'Éphrem ne porte pas spécifiquement sur le débat autour des incorporels et, par conséquent, ne semble pas vouloir résumer l'écrit d'Albinus. Étant donné qu'Éphrem ne connaît Albinus qu'à travers la polémique de Bardesane, il est possible qu'il cherche seulement à justifier le fait qu'un platonicien aborde une question stoïcienne, car Bardesane semble le lui reprocher ${ }^{11}$. En outre, l'appréciation de l'enchaînement de l'argumentation d'Albinus, platonicienne et stoïcienne, contraste avec la critique de l'attitude de Bardesane qui est décrit plus loin comme « incapable de parler » et ignorant des doctrines de Platon.

Sur le contenu de l'écrit de Bardesane, un témoignage inattendu vient peut-être de Serge de Reš'ayna. Celui-ci écrit, en commentant les Catégories d'Aristote : « Mais si quelques-uns des stoïciens, qui pensent qu'il n'y a rien qui ne soit corporel, et avec lesquels s'accorde, dans l'argument ( $\left.s^{\prime} a r b \bar{a}\right)$ des qualités, aussi le Syrien Bar Daysan, ont cru que les (qualités) aussi ... sont des corps $^{12}$. $>$ Dans le commentaire de ce passage, G. Furlani explique que le terme « argument » $\left(\check{s}^{2} a r b \bar{a}\right)$ peut être traduit par «traité »; cependant, il refuse à Bardesane la capacité de rédiger un écrit de ce type, parce qu'il «n'était pas

rapprochements avec Éphrem ; voir Beck 1976 p. 32 n. 25.

9. Voir Orth 1947 et 1958. L'impossibilité de cette attribution a été démontrée par Giusta (éd.) 1976.

10. Voir Bréhier 1997 [1907] p. 2.

11. L'auteure remercie Thomas Bénatouil de lui avoir suggéré que la méthode des sages et philosophes évoquée par Éphrem ressemble à la méthode préconisée pour l'enseignement par Chrysippe (voir De stoic. rep. $1035 \mathrm{f}=$ SVF II, $127=L S 31 \mathrm{P}$ ).

12. Le fragment $47 \mathrm{c}$ du Traité sur les Catégories d'Aristote, publié et commenté par G. Furlani - voir Furlani 1937, p. 349-350. 
un philosophe pur ou un commentateur des œuvres d'Aristote $\gg^{13}$. Grâce au témoignage d'Éphrem, nous savons que dans le discours De Domnus, Bardesane parle aussi des qualités des incorporels (fragments 16 et 17) ; il n'est donc pas impossible que Serge de Reš'ayna fasse allusion à ce texte-là ${ }^{14}$. Cette information est la seule que Serge retient du texte de Bardesane. Son témoignage fait penser que la lecture d'Éphrem, quoique bien plus complète, pourrait être tout aussi sélective, et que le débat de Bardesane avec Albinus aurait porté sur des questions de physique, de théologie et de logique faisant appel à la doctrine des incorporels.

Le début du fragment 2 correspond à l'endroit où le copiste a indiqué un nouveau paragraphe. Éphrem cite encore la phrase de Bardesane du fragment 1, à laquelle il ajoute que « les stoöciens disent la même chose »; ensuite, il cherche dans son commentaire à expliquer les différents sens accordés aux termes « corporel » / « incorporel » par les uns et les autres. L'explication s'articule autour de trois affirmations dont il convient de vérifier l'exactitude :

- selon les platoniciens, « les corporels et les incorporels existent en nature et en substance $\gg$;

- selon les stoïciens, « tout ce qui existe en nature et en substance est un corps »;

- « incorporel » est « ce qui est conçu dans l'intellect », sans pourtant exister $\ll$ en nature $\gg$.

La première affirmation est très approximative. Platon parle très rarement des $\dot{\alpha} \sigma \omega ́ \mu \alpha \tau \alpha$ et sans donner à ce terme une connotation spécifique ${ }^{15}$. En revanche, pour élaborer la théorie des idées, il parle des von đó (intelli-

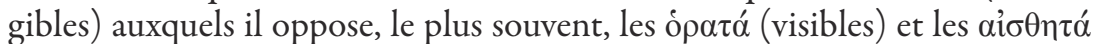
(sensibles) lorsqu'il parle de la connaissance ${ }^{16}$. De manière générale, pour les

13. Furlani 1937, p. 352. Le sens du terme šarbā est très large et peut vouloir dire histoire ou discours, oral ou écrit.

14. Plus loin, il évoque des qualités, telles la couleur, la figure et la forme, qu'on retrouve aussi dans le discours éphremien, en tant que sujets de réflexion de Bardesane ; cf. fragment 16 et 17. Il n'y a pas d'autre témoignage ni fragment de Bardesane concernant les qualités des incorporels.

15. Platon parle du discours achevé comme « une sorte de monde incorporel » (Philébe, 64b) ou encore de l'harmonie du corps humain - celle de la lyre et de la corde comme « quelque chose d'incorporel ... divin » (Phédon, 85e). Dans le Sophiste, il s'oppose à la théorie des penseurs qui n'admettaient que l'existence des corps (Sophiste, 246b). L'incorporel apparaît aussi dans le Timée, 27d-29d, dans l'explication de la formation du monde. Dans le Timée (39c-31a; 36e-37a; 39de; 48e-49a; 92c), Platon parle des « vivants intelligibles » (les dieux

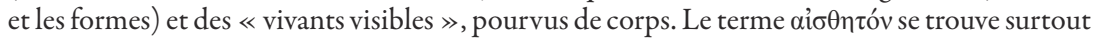
dans le Théététe $(156 \mathrm{bc} ; 182 ; 184)$, dans les passages concernant la connaissance sensible.

16. Cf. République, VI, 508b-511c (analogies du soleil et de la ligne) ; République, VII, 517 (allégorie de la caverne). Dans le Phédon, 82d-83c, les choses perçues par l'âme sont « sensibles et visibles » et « sensibles et intelligibles ». 
platoniciens, les « incorporels » seraient non seulement les idées, mais aussi les dieux, les démons, les âmes et la matière première. Les médio-platoniciens

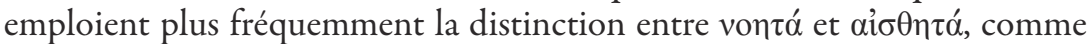
le remarquent aussi les commentateurs du Discours éphremien ${ }^{17}$. En tant que sujet traité à part, la question des incorporels paraît plutôt marginale dans la physique platonicienne ${ }^{18}$, mais la notion d'incorporel peut néanmoins apparaître dans d'autres contextes, surtout lors de réflexions sur l'être et la matière. Les exemples d'une telle attitude nous viennent de plusieurs auteurs. Dans le traité Sur le Bien, Numénius étudie les notions de corps et de matière et, à cette occasion, il dit que l'être est incorporel et qu' il est intelligible ( $\tau$ ò

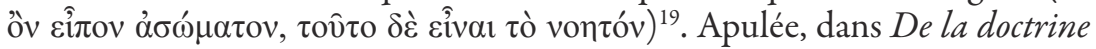
de Platon, lorsqu'il étudie Dieu, la matière et les idées, affirme que Dieu et les idées sont incorporels, tandis que la matière n'est ni corporelle ni incorporelle ${ }^{20}$. Croyant qu'il s'agit d'une ouvre d'Albinus, E. Beck, dans son commentaire du Discours contre le «De Domnus », évoque le Didaskalikos ${ }^{21}$.

17. Cf. Beck 1976 p. 29, 30, 2. Sur cette distinction chez les auteurs médioplatoniciens (Philon d'Alexandrie, Timée de Locres, Arius Didyme, Plutarque, Numénius, Apulée, Clément d'Alexandrie), voir Dörrie \& Baltes (éd.), 1993, p. 280-289. Voir aussi Sorabji 1988. U. Possekel donne des références à Philon d'Alexandrie, De agric. 139, Clément d'Alexandrie, Stromata, 8, 20 et Grégoire de Nysse, Hom. Opif. 8, 5 ; cf. Possekel 1999 p. 160-161. Sur

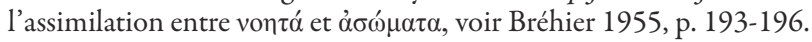

18. Sur ce sujet, il y a un écrit du Pseudo-Galien, Que les qualités sont incorporelles et deux traités de Plotin, Sur la qualité, II, 6 [17] et Sur l'impassibilité des incorporels, III, 6 [26]. Deux autres écrits Sur les incorporels, connus seulement par leurs titres, seraient celui d'Albinus mentionné uniquement par Éphrem - et celui de Porphyre ; voir Dörrie \& Baltes (éd.) 1993 p. 280. Voir aussi Todd 1977.

19. Fr. $4 \mathrm{a} ; 4 \mathrm{~b} ; 6 ; 7$. Dans les fragments 6 et 7, Numénius affirme que l'être est incorporel et intelligible - voir Des Places (éd.) 1973 p. $49-50$.

20. Apulée, Platon et sa doctrine, I, 190-192; voir Beaujeu (éd.) 1973 p. 65. Le lien entre Apulée et l'auteur du Didaskalikos a été démontré en 1905 par T. Sinko qui voit des liens entre Apulée, Albinus et Galien (voir Sinko 1905). Ce travail est au fondement du chapitre 6 (« Albinus, Apuleius, Galen ») du livre de J. Dillon qui attribue le Didaskalikos à Albinus ; voir Dillon 1977 p. 266-340 et 419. Dillon change d'avis dans son édition d'Alcinoos (Dillon, trad., 1993). De manière générale, les travaux sur l'entourage de Galien favorisent les comparaisons avec d'autres médio-platoniciens, aussi bien grecs que latins, ainsi qu'avec des idées aristotéliciennes et stoïciennes - voir l'introduction de Dillon (trad.) 1993 p. XVIIXXII et XXX-XL. Voir aussi Göransson 1995. Sur les médioplatoniciens latins, voir Gersh 1986 p. 197-214 (Aulu-Gelle) et p. 215-328 (Apulée).

21. Beck cite des passages du Didaskalikos - dont il suit l'attribution à Albinus et de Plotin, Enn. II, 4, 1 ; voir Beck 1976 p. 29-31. Pour reconstituer l'œuvre d'Albinus, J. Freudenthal a proposé en 1879 de lire le nom d'Alcinoos, l'auteur peu connu du Didaskalikos, comme une variante paléographique d'Albinus ; cette hypothèse figure dans les publications anciennes - voir Witt 1937 et la traduction du chapitre X du Didaskalikos attribuée à Albinus par Festugière 1954, p. 95-102. Le nom d'Albinus comme auteur du Didaskalikos figure dans l'édition de Louis (éd.) 1945 et celle de Invernizzi (éd.) 1976. Ce n'est plus le cas de la nouvelle édition et traduction française de Whittaker 1990. Dans son article sur le Discours contre le "De Domnus", E. Beck cite le Didaskalikos comme une œuvre d'Albinus - voir Beck 1976 
En effet, plusieurs passages du Didaskalikos peuvent servir de référence sur la question de l'incorporel : la distinction entre l'intelligible et le sensible (chap. IV), mais surtout un long développement sur les qualités et les causes en tant qu' incorporels ${ }^{22}$.

La lecture des fragments du Discours contre le «De Domnus » fait penser qu'Éphrem emploie le terme « incorporel » (lä gšom) dans sa connotation médio-platonicienne, pour désigner les différentes catégories d'êtres

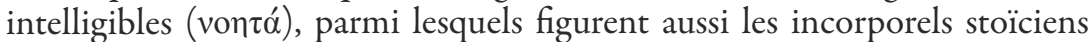
$(\dot{\alpha} \sigma \omega ́ \mu \alpha \tau \alpha)$. Le terme syriaque qu'il utilise est suffisamment général pour le permettre. Cependant, tout porte à croire que le platonisme d'Éphrem résulte d'une alliance circonstancielle ayant pour but de combattre le matérialisme stoïcien de Bardesane, comme nous allons le démontrer au fur et à mesure de notre étude des fragments du Discours.

Quelle était la compréhension de la doctrine stoïcienne par Éphrem ? À la différence des platoniciens, les stoïciens considèrent comme des corps la matière première, l'âme, le dieu, les qualités et les vertus. En général, la

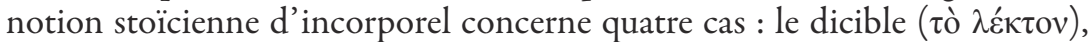

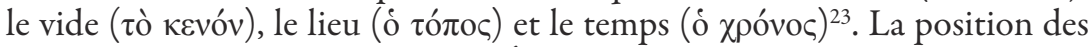
stoïciens a certainement influencé Éphrem - via Bardesane - car il consacre les parties successives de son traité à l'examen de ces notions. Il cite également la phrase : « tout ce qui existe est un corps », qui est une opinion commune au sujet de la doctrine stoïcienne ${ }^{24}$. En revanche, la définition des incorporels

p. 29 n. 17 et p. 30 n. 19. Sur la distinction entre Albinus et Alcinoos, cf. Giusta 1960-1961 ; Whittaker 1974 p. 320-354 et p. 450-456. Néanmoins, le débat continue : voir Mazzarelli 1980. Quant à la référence à Plotin, signalée également par E. Beck, rien ne permet de la confirmer - voir Beck 1976 p. 31, n. 24. Possekel ne relève aucun trait plotinien chez Éphrem - voir Possekel 1999 p. 155-185.

22. Didaskalikos, XI, 15-37. La doctrine exposée dans ce chapitre ressemble à celle d'Alexandre d'Aphrodise dans le De anima mantissa, 123. Le chapitre sur les incorporels a servi de fondement à la comparaison avec le Que les qualités sont incorporelles du PseudoGalien - voir Whittaker 1974 p. 450 et Moraux 1984, p. 470-472.

23. Voir SVF II, 331; Bréhier 1997 [1907]. L'incorporel est défini chez Sextus Empiricus comme ce qui n'est pas capable d'agir et de pâtir ( $M$. VIII, 263) et réparti en quatre classes d'incorporels : l'exprimable, le lieu, le vide et le temps (M.X, 218) - voir aussi Plutarque, Sur les notions communes contre les stö̈ciens, 1073d10-1074d11. E. Beck indique la ressemblance entre le Discours contre le 'De Domnus' et les traités de Sextus Empiricus, ce qui ne prouve pas grand chose, car Sextus est notre principale source d'information sur la théorie stoïcienne des incorporels.

24. La conception stoïcienne du corps est mentionnée par plusieurs auteurs, pas nécessairement stoïciens : le corps est défini comme ce qui possède une triple dimension, en longueur, en largeur et en hauteur (Diogène Laërce, VII, 135), et ce qui agit et pâtit (Cicéron, Acad. Post. I, 39; Plutarque, Sur les notions communes contre les stoïciens, 1073d10-1074d11). L'affirmation rapportée par Éphrem ressemble à un passage du livre VII de Diogène Laërce (VII, 150) : «Ils disent que la substance de tous les êtres est la matière première (...) Selon eux, la substance est un corps et elle est limitée (...) » 
stoïciens comme « ce qui n'existe pas par nature, mais peut être conçu par l'intellect » (metrgeš b-ré yānā) semble provenir d'Éphrem et elle est problématique. En effet « ce qui est conçu dans l'intellect » pour un stoïcien peut désigner plusieurs choses, dont toutes ne sont pas des incorporels ${ }^{25}$. La liste des interprétations possibles de la formule éphremienne est longue, car on peut penser au $\lambda \varepsilon \kappa \tau$ óv (dicible, exprimable) - « ce qui subsiste en

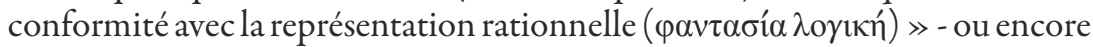

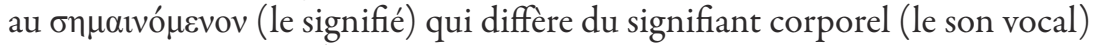
et de l'objet corporel ${ }^{26}$. Ainsi l'expression « conçu par l'intellect » n'atteste pas une compréhension correcte de la doctrine stö̈cienne par Éphrem.

En revanche, «ce qui est conçu par l'intellect » répond davantage aux caractéristiques du von đóv platonicien. Apulée utilise une formulation semblable, lorsqu' il écrit « ce qui n'a pas une substance corporelle n'est perçu que par la pensée $\gg^{27}$. Selon plusieurs témoignages, les stoïciens disaient que les idées platoniciennes ne sont qu'un pur fantasme de l'âme ('̇vvoń $\mu \alpha \tau \alpha$

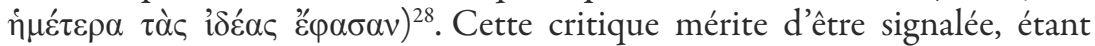
donné que la polémique entre les platoniciens et les stoïciens constitue l'arrière-plan du traité d'Éphrem.

Aux « incorporels » ainsi définis, Éphrem oppose « ce qui existe par nature et par substance ». Que veut-il dire par là ? L'expression syriaque

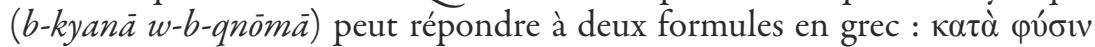

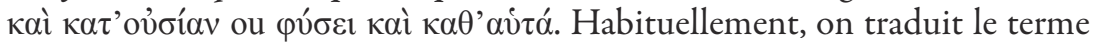

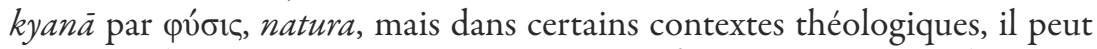
correspondre aussi aux termes essentia et substantia ${ }^{29}$. Le sens du terme qnōmà est particulièrement large et il peut être traduit par plusieurs termes

25. Les stoïciens ont opéré une distinction entre l'objet de la représentation mentale ( $\varphi \alpha v \tau \alpha \sigma i ́ \alpha)$ et la représentation elle-même. Sur la $\varphi \alpha v \tau \alpha \sigma i ́ \alpha$ stoïcienne, voir SVF I 59-70. Les témoignages les plus proches du texte éphremien sont ceux de Diogène Laërce VII, 50 et 51 (SVF I, 60 et 61) et de Sextus Empiricus M. VII, 241 (SVF I, 64). Voir aussi Chiesa 1991, et Gourinat 2013.

26. La plus longue description des représentations vient de Sextus Empiricus, $M$. VII, 242 (SVF I 65). Elle est citée par Beck 1976 p. 32, n. 25. Sur le signifié, le signifiant et l'objet corporel, voir aussi Sextus Empiricus, M. VIII, 409 (SVF II 85). Diogène Laërce rapporte, entre autres, la position de Chrysippe pour qui les représentations sont à comprendre de manière métaphorique (VII, 49-51; SVF II, 520).

27. Sed quae substantiam non habent corporum, ea cogitationibus uideri; Apulée Plat. I, 192 dans Beaujeu (éd.) 1973.

28. La critique des idées des platoniciens a été formulée par Zénon et reprise par les adeptes de son école ; voir SVF I, 494 (Syrianus, In Metaph. 892) ; SVF II, 360 (Aëtius). Une étude des témoignages transmis par la tradition doxographique se trouve chez Dörrie \& Baltes (éd.) 1993 p. 14-18 (texte) et p. 232-234. Sur les idées platoniciennes comme « incorporels », voir Baltes 1996.

29. Thesaurus syriacus, col. 1703-1705. 


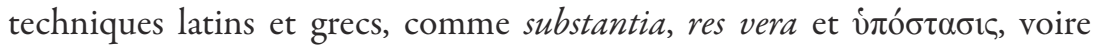
même persona et individuum ${ }^{30}$. Cependant, le fait d'associer kyanā et qnōmā plaide en faveur d'une traduction par les termes « nature » et « substance », entendus comme effort pour mettre en valeur l'existence concrète, corporelle, de certaines catégories d'êtres, autres que les « incorporels ». Notons que dans la suite du Discours, le terme qnōmā (ov̉oía) paraît avoir une signification proche de gušm $\bar{a}(\sigma \omega ́ \mu \alpha)^{31}$. Cette terminologie est donc plutôt éphremienne que stö̈cienne ${ }^{32}$.

Dans le fragment 3, Éphrem caractérise la position de Bardesane qui, selon lui, s'est opposé à la doctrine platonicienne, mais sans la connaître suffisamment. D’après Éphrem, la critique bardesanite portait sur les idées appartenant à d'《 autres »- sans doute d'autres philosophes - que Platon et ses disciples. Mais qui sont ces « autres », combattus par Platon ? D'un côté, il est vrai que les premiers adversaires de Platon étaient des « physiciens », des philosophes de la nature, et que la physique stoïcienne a quelques traits communs avec leurs doctrines. Mais d'un autre côté, rien n'indique qu'Éphrem ait étudié Platon. Son but est de combattre Bardesane et non pas de défendre le platonisme - qui, d'ailleurs, n'est plus mentionné dans le Discours ${ }^{33}$. Ainsi le stoïcisme défendu par Bardesane doit-il se présenter à Éphrem comme une doctrine « physique » et matérialiste, dont les contradictions apparaissent à l'évidence dans la théorie des incorporels. Mais les frontières entre le platonisme et le stoïcisme dans le Discours sont brouillées par le fait que Bardesane n'est pas conséquent dans son adhésion au Portique, notamment lorsqu'il professe une doctrine des « êtres-éléments », dont Dieu et la Parole de Dieu font parfois partie ${ }^{34}$. Par conséquent, Éphrem entre dans le débat entre les platoniciens et les stoïciens pour combattre la théologie de Bardesane, et la théorie des incorporels l'intéresse dans la mesure où elle s'inscrit dans son projet théologique.

30. Thesaurus syriacus, col. 3667-3668.

31. La même expression revient plus loin : 10, $48 ; 11,47 ; 12,20 ; 13,48 ; 14,20 ; 17,7$. Voir Beck 1976, p. 31-32, n. 24.

32. Pour les stoïciens la matière est maintenue ensemble par la force interne (๕๕६ıৎ), qu'on

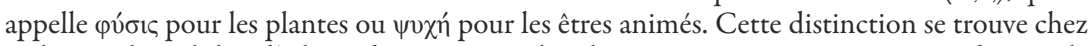
Galien et chez Philon d'Alexandrie - voir Sambursky 1959, p. 7-11. Quant au second terme, la substance (ovoí $\alpha$ ), les stoïciens appellent ainsi la matière première sans qualité - voir Diogène Laërce, VII, 150. Voir aussi la critique de Plotin, Ennéades, II, 4 [12] : « Les uns admettent que les corps sont les seules réalités et la seule substance. »

33. Éphrem parle encore de Platon et de sa doctrine quand il aborde le manichéisme (Contre Mani dans Bevan \& Burkitt (éd.) 1921, 209, 27-30 et 211, 13-15). Dans les Carmina Nisibena, il mentionne les stoïciens, les platoniciens et les cyniques, mais il observe aussi que les noms des écoles philosophiques viennent de leurs fondateurs - voir Carm. Nis. 25, 9-16. Voir Possekel 1999 p. 54.

34. Voir Beck 1978. 


\section{Définition stö̈cienne de l'incorporel (fr. 4)}

Fr. $4\left(9,20-32^{35}\right)$

... qui <sont > incorporels, ... « qui n'ont pas trois dimensions (tlätā diasțtata,

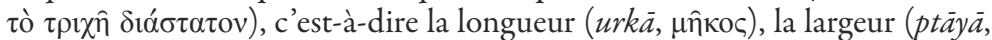

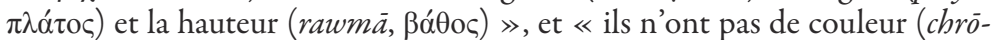
$m a, \chi \rho \hat{\omega} \mu \alpha)$ non plus et ... que nous considérons ... » et « le temps et le lieu

... la ligne $($ surțā), la longueur, la largeur ... qui sont connus. »

Il est impossible d'inscrire le fragment 4 dans une section thématique, car il provient d'une partie très endommagée du texte. Nous le mentionnons malgré son état fragmentaire parce que le terme lam y figure à trois reprises ; il s'agit donc d'une citation tirée de l'écrit de Bardesane qui nous renseigne sur le contenu de la polémique. Apparemment, Éphrem a choisi de transcrire des passages qui sembleraient être une définition stoïcienne des incorporels, comme on le constate à partir des nombreuses translittérations des termes grecs $^{36}$. Certains stoïciens donnent une description géométrique des corps, en

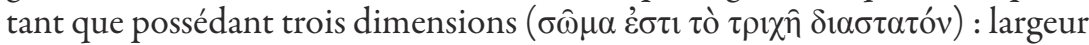

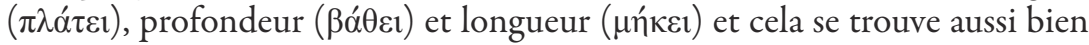
chez Chrysippe que dans la doxographie stoïcienne ${ }^{37}$. Cependant, dans la suite du texte, on perçoit le mot « lieu » qui est un incorporel stoïcien et qui possède les trois dimensions mentionnées dans le fragment. Par conséquent, même si l'état du texte ne permet pas de préciser si Éphrem cite les opinions de Bardesane lui-même ou les opinions de quelqu'un d'autre rapportées par Bardesane, on peut admettre que la citation relate la position stoïcienne sur les incorporels.

\section{Le soleil et la lumière (fr. 5 et fr. 19)}

Fr. $5\left(9,33-10,10^{38}\right)$

$<$ Cependant $>$ si quelqu'un dit, au sujet du soleil, qu'il est mortel, ceci ... c'est à cause de la circonférence (eskemā - $\chi \chi \hat{\eta} \mu \alpha)$ du soleil, telle qu'on la voit. Il a dit à ce sujet qu'il « est né en Orient et qu'il ... dans le Midi et qu'il arrive et étend <ses rayons > jusqu'en Occident, ... et il a appelé le soleil mortel. Il s'est précipité à blâmer, <tandis que> celui qui a blâmé est, lui-même, combien plus blâmable. » Quant au fait que les stoïciens ont désigné ainsi ces choses

35. De ce passage très lacunaire, Beck conserve seulement une ligne $(9,28)$ dans le fr. VII $(8,15-10,10)$.

36. Le texte éphremien rappelle ceux de Sextus Empiricus (M. VIII, 24-25), de Plutarque (Sur les notions communes contre les stö̈ciens, 1073d10-1074d11) ou encore du Pseudo-Galien (Que les qualités sont incorporelles, 2).

37. SVF II, 537, 538 ; il s'agit de fragments de Chrysippe transmis par Arius Didyme et par Philon d'Alexandrie. Voir aussi SVF III, 6 (Apollodore).

38. Toujours à cause de lacunes, E. Beck se limite à conserver seulement la dernière phrase $(10,4$ s.) dans le fr. VII $(8,15-10,10)$. 
qui < sont $>$ incorporelles ... <c'est > moi qui suis en train de dire comment et pour quelle raison, et en vue de quoi.

Fr. 19 (39, 48-40, 12) ; Beck, fr. XXXII $(39,6-40,12)$

La lumière $(n u b r \bar{a})$ ne peut rien absorber, parce que sa nature $(k y a n \bar{a})$ dense ... Elle n'a pas absorbé l'obscurité ; l'obscurité est complètement détruite et disparue, parce qu'il n'y avait dans le ciel rien qui soit incorporel...

Pour comprendre de quoi parle le fragment 5, fortement endommagé, on peut lui ajouter le fragment 19 , qui vient de la section finale du Discours, consacrée à la description des mécanismes de la vue, de la lumière du soleil et de la lune. En réalité, les deux fragments dépendent des conceptions bardesanite et éphremienne du soleil et de la lumière.

Le fragment 5 parle d'un «blasphème » qui consiste à dire que le soleil est mortel. On comprend mal à quoi se réfère la dernière phrase disant que « les stoïciens ont appelé ces choses incorporelles ( $l \bar{a}$ gšom) », mais il s'agit probablement des astres. En effet, selon les stoïciens, le soleil, étant fait de feu, est corporel et vivant ; comme les autres corps célestes, il se nourrit des émanations de l'eau ou de la terre ${ }^{39}$. Son éventuelle mortalité n'a pas besoin d'être explicitement formulée, parce qu'elle est sous-entendue dans la théorie de la conflagration finale ${ }^{40}$. Stobée (ou plutôt Arius Didyme) a conservé un fragment, où Chrysippe parle aussi du « lever » et du « coucher »du Soleil ${ }^{41}$ - et cette même idée figure dans le fragment 5 . On peut alors imaginer qu'Éphrem critique l'idée stoïcienne, selon laquelle les corps célestes sont corporels (donc mortels) et qu'il l'attribue à une compréhension naïve des mouvements du soleil qui « naît » en Orient et « se couche » en Occident. Le sens habituel du terme eskemā, que nous traduisons par « circonférence », est celui de « figure », voire « figure géométrique ». Dans le cas précis du soleil, sa « figure » serait une ellipse de la route qu'il parcourt dans le ciel au long d'une journée. En partant de cette observation, Éphrem se déclare prêt à expliquer comment il faut comprendre ces phénomènes.

Il est hors de doute qu'Éphrem émet des réserves par rapport à la doctrine qui considère le soleil comme un corps de substance ignée. Heureusement, nous disposons d'un texte où Éphrem parle du soleil, en dehors du débat sur les incorporels. Notre passage provient du troisième Discours à Hypatios, faisant partie du même recueil hérésiologique que le Discours contre le «De Domnus » :

39. On attribue à Chrysippe l'idée que le soleil est une masse ou un « flambeau

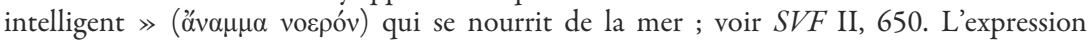
« flambeau intelligent » vient d'Héraclite A 12 DK.

40. Le soleil est une masse ignée ; voir $S V F$ II, 650, 652, 655, 656. Au sujet de la conflagration qui suppose la mortalité du soleil, on peut consulter $S V F$ II, 585-595.

41. SVF II, 683. Le même fragment parle des astres qui se lèvent et se couchent avec le soleil. Sur les astres, voir surtout $S V F$ II, 681, 682, 686, 688 . 
Le soleil est une chose et son éclat $\left(\operatorname{denh}_{\bar{a}}\right)$ est en une autre. Le soleil possède une substance et une limite (hudrā); et l'œil peut le délimiter. Mais son éclat n'a ni limite $(s k \bar{a})$ ni substance $(q n \bar{m} m \bar{a})$; et l'œil ne peut pas le délimiter. ... Parce que le soleil est feu (nurā), nous apprenons à le connaître grâce à un feu inférieur, <à savoir > la flamme du feu qui possède la substance (qnōmā) ; <en revanche> la lumière $(n u h r a \bar{a})$ du feu n'a pas de substance. Les corps (pagrēe vont et viennent à l'intérieur de la lumière sans obstacle, mais les corps (gušamè) ne peuvent pas s'approcher de la substance (du feu $)^{42}$.

Le passage cité montre qu'Éphrem distingue entre le soleil corporel, fait d'une substance ignée, et la lumière qui n'a pas de substance. Quelle que soit son intention, par sa critique de l'idée du soleil comme mortel, Éphrem se rapproche du platonisme. Pour Platon et ses disciples, le soleil et les astres sont des êtres vivants d'espèce divine - donc immortels - dont les corps sont faits de feu ${ }^{43}$.

Cette même problématique intervient dans le fragment 19 qui, toutefois, semble rendre compte de la position de Bardesane. Dans un autre écrit antibardesanite du même recueil hérésiologique, Éphrem accuse Bardesane d'enseigner que la lumière et les ténèbres sont corporelles ${ }^{44}$. Il s'agit donc d'une polémique sur les conceptions cosmologiques de Bardesane, selon qui l'univers est composé des ìtyē (êtres-éléments) ou estuksē ( $\sigma \tau 0 \imath \chi \varepsilon \hat{\imath} \alpha)$, parmi lesquels figurent la lumière et les ténèbres. Un écho de cette critique revient dans le fragment 19 , où la lumière est « dense » et où la disparition des ténèbres prouve l'absence des incorporels dans les cieux.

\title{
4. Le lieu comme incorporel (fr. 6-12): un lieu dans un autre
}

\begin{abstract}
Fr. $6(10,18-11,7)$; Beck, fr. VIII $(10,11-12,18)$
... ceux-là sont des noms (šmāhēe) appliqués aux corps (b-gušmēe et aux substances $(b$-qnōmēe). Et ils ont commencé à parler au sujet du lieu (atrā); donc ce lieu-là existe en nom $\left(b-s_{m} m \bar{a}\right)$ et en signification $(b$-sukkāla $)$, comme je l'ai dit. À cause de son nom, il est exprimé par la voix (b-qālā) et à cause de sa signification il est conçu par l'intellect (b-re 'yānāa). Et si toi, ô auditeur, tu n'écoutes pas avec l'intellect, tu ne pourras pas l'entendre.

Regarde donc : en ce qui concerne le lieu, il résulte, nécessairement, qu'il existe et qu'il n'existe pas. Et si ces deux < possibilités> ne sont pas, le lieu ne peut ni exister, ni recevoir un nom. Cela veut dire qu'il existe en nom $<$ seulement $>$, tandis qu'il n'a ni corps ni substance. Toutes choses alors,
\end{abstract}

42. Éphrem, Discours à Hypatios, III, dans Mitchell (éd.) 1912, 50, 40-51, 28.

43. Voir Platon, Timée, 40a ; Les Lois, X, 898d. Dans le Didaskalikos, le soleil est appelé

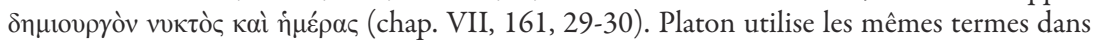
le Timée pour parler de la terre (40c1-2) ; voir Whittaker 1989b, p. 63-95. L'exemple est mentionné dans l'édition du Didaskalikos, p. xxviii-xxix et p. 94. Voir Donini 1988.

44. Voir Stances contre Bardesane dans Bevan \& Burkitt (éd.) 1921 p. 48-49 et p. 60-63. 
fussent-elles substance ou corps, ne peuvent exister en celui-ci, car il est incorporel. »

Fr. 7 (12, 3-12, 18); Beck, fr. VIII $(10,11-12,18)$

Qu'on revienne encore sur sa vérité : celle qui consiste à dire que $<$ le lieu $>$ n'est pas corporel et que pour cette raison il n'est pas dans un espace $($ dukta $)$ - c'est ainsi que les stoïciens l'ont dit. Voici donc ce lieu qui s'installe à l'intérieur d'un lieu ! < Mais> il n'est pas possible qu'un lieu s'installe à l'intérieur d'un lieu, sinon tout ce qui a été dit auparavant serait invalidé.

Fr. $8(12,19-12,32)$; Beck, fr. IX $(12,19-13,46)$

$\mathrm{Si}$ on trouvait un certain lieu corporel (atrā gš̀ima), possédant une substance, et si on trouvait un autre lieu incorporel (atrā $\left.d-l \bar{a}-g s^{\circ} o m\right)$, alors il serait possible <que> le corporel soit installé à l'intérieur de ce qui est incorporel. Je suis en train de parler de choses que l'on peut exprimer, mais qui ne peuvent exister, je parle donc avec audace.

Les fragments concernant le lieu comme incorporel sont très nombreux (6 à 12), parce qu'Éphrem discute les différents aspects de cette question. Les fragments 6, 7 et 8 expriment la même objection : les stö̈ciens prétendent qu'un lieu existe à l'intérieur d'un autre lieu. Pour introduire cet argument, Éphrem complète la définition de l'incorporel, telle que nous l'avons vue dans le fragment 2 , où l'incorporel est défini comme «ce qui peut être conçu par l'intellect ». Au début du fragment 6, l'incorporel est aussi « ce qui existe en nom et en signification ». Il peut être exprimé par la voix à cause de son nom et conçu par la pensée à cause d'une certaine compréhension ${ }^{45}$. Pour traduire le terme sukkālā, qui veut dire « le signifié » ou « le sens », nous avons choisi l'expression générale «signification » qui nous paraît plus proche de la manière dont Éphrem définit l'incorporel comme « ce qui est conçu par l'intellect ». Nous aurons plus loin l'occasion d'en dire davantage, les notions de nom et de signification étant abondamment discutées dans les fragments 12 et 16 ; mais ici Éphrem ne fait que les évoquer rapidement pour dire qu'un lieu incorporel est impossible.

Dans la conception stoïcienne, le lieu est ce qui est occupé entièrement par un corps, ce qui coïncide avec l'idée d'extension tridimensionnelle ${ }^{46}$. D'une certaine façon, le lieu est toujours plein, parce qu'il est constitué par

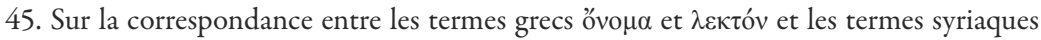
šmà et sukkālāa, voir l'analyse de Beck 1976, p. 33-34 n. 31. Cependant, le sens de sukkâlā semble être plus large que celui de $\lambda \varepsilon \kappa \tau$ óv; voir les fragments 12 et 16.

46. «Zénon et ses disciples distinguent entre le vide, le lieu et l'espace : le vide est l'absence de corps, le lieu est ce qui est occupé par un corps et l'espace est ce qui est partiellement rempli », SVF I, 95 ; voir aussi : SVF II, 162, 501, 504, 505. Sur le concept stoïcien de lieu, voir Algra 1995, p. 261-334. Bréhier 1997 [1907] partage l'hypothèse d'une inspiration aristotélicienne dans la doctrine du Portique, fondée sur des informations venant de commentateurs d'Aristote comme Thémistius et Simplicius (p. 37-53). Cette opinion a été remise en question et affinée par Algra 1995, p. 336-339. 
le corps qui l'occupe ou qui peut l'occuper. Pour parler du lieu, les stoïciens utilisent donc une série de concepts étroitement liés à cette définition, à

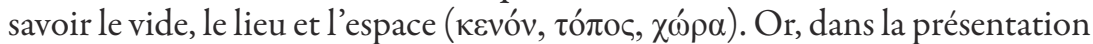
de cette doctrine par Éphrem, figurent seulement deux de ces notions : le lieu et l'espace.

Dans son commentaire du Discours, Beck observe une ressemblance entre l'argumentation éphremienne et l'examen critique du concept stoïcien de lieu par Sextus Empiricus. Sextus dit notamment, à propos du lieu qui à la fois existe et n'existe pas (voir frag. 6), qu'un lieu ne peut pas exister à l'intérieur d'un autre lieu (voir frag. 7) et qu'il est impossible qu'un lieu corporel soit à l'intérieur d'un lieu incorporel (voir frag. 8$)^{47}$. Ceci dit, il saute aux yeux que l'argumentation d'Éphrem est considérablement plus simple. Peut-être est-ce lié au vocabulaire qu'il emploie. Pour désigner le lieu, Éphrem utilise le terme atrā qui correspond à $\tau$ ó $\pi$ oc. Dans le fragment 7 , figure un autre terme, duktā, qui en syriaque possède un sens très large : « espace, endroit,

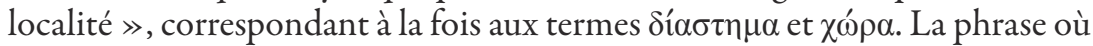
il figure est attribuée aux stoïciens, mais le commentaire qui la suit montre que le terme duktā n'est pas forcément compris au sens stoïcien. Parce que la notion des limites du lieu est abordée dans les fragments suivants, on admet que duktā veut dire $\chi \omega ́ \rho \alpha$. Or, la $\chi \omega ́ \rho \alpha$ stoïcienne est une étendue occupée par les corps et par le vide, mais elle est parfois comprise comme une sorte de $\tau$ ó le fragment 7. Mais l'explication qui suit dans le fragment 8 est différente : Éphrem parle d'emblée d'un lieu « corporel », doté d'une substance. Ce lieu aurait pu être installé à l'intérieur d'un lieu « incorporel » - à condition qu'on veuille admettre l'existence d'un lieu incorporel. En disant cela, Éphrem semble être influencé par la conception platonicienne de la $\chi \omega ́ \rho \alpha$, comme milieu spatial qui reçoit les images des Idées, devenant ainsi la « nourrice » des choses sensibles qui sont les copies des Idées ${ }^{49}$. Le statut ontologique de la $\chi \omega ́ \rho \alpha$ est difficile à préciser ; c'est un espace actif et une matrice porteuse de toute matière ${ }^{50}$. Même si le platonisme n'est pas explicitement évoqué, Éphrem paraît reproduire une argumentation d'inspiration platonicienne. Il s'avère, une fois de plus, que la critique éphremienne utilise des arguments platoniciens contre le stoïcisme de Bardesane.

Le fragment 8 qui conclut à l'impossibilité de faire coïncider un lieu incorporel avec des corps donne raison à la conception platonicienne. Cette

47. Cf. Beck 1976, p. 34. Ces trois démonstrations figurent chez Sextus ; voir $P$. III, 131 (frag. 6) ; III, 129 (frag. 7) ; III, 134 (frag. 8).

48. Sur les différentes conceptions de la $\chi \omega ́ \rho \alpha$, notamment celles relayées par Sextus Empiricus qui présente la $\chi \omega ́ \rho \alpha$ comme un genre de tó

49. Voir le chapitre « Milieu spatial » dans Brisson 1998 p. 175-266.

50. Voir Platon, Timée, 47e-52d et Sambursky 1977, Algra 1995 p. 33-38 et p. 72-120. 
argumentation paraît très simplifiée, mais le fragment suivant en explique la raison.

\section{Le lieu comme incorporel (fr. 9) : une question théologique}

Fr. $9(13,27-13,46)$; Beck, fr. IX $(12,19-13,46)$

... aucun autre lieu ne peut exister à côté de celui-ci, parce que le ciel et la terre sont advenus au milieu des choses créées qui existent à l'intérieur du monde habité, les limites (puršānēe du monde sont appelés lieux (atrwātā), qu'ils soient délimités ou excédants. Ceux-là sont des noms de pays et de lieux habités ; le lieu (atrā) qui se trouve alors au milieu de ces lieux, celui-là dont nous parlons, il est incorporel.

Les expressions utilisées dans ce fragment sont de portée théologique. Le fragment 9 parle de la création du ciel et de la terre qui apparaissent au milieu des choses créées. Le mot « limites », puršānēe, vient de la racine prš qui signifie « séparer, faire différence » et il correspond à de nombreux termes grecs $(\delta 1 \alpha \sigma \tau o \lambda \eta ́, \mu \varepsilon \rho ı \sigma \mu o ́ c, \chi \omega \rho ı \sigma \mu o ́ \varsigma)^{51}$. Ici, Éphrem ne s’inspire pas de la philosophie, mais de la Bible. Dans le livre de la Genèse (I, 4-6), Dieu fixe les limites de l'univers en séparant (prś) la lumière des ténèbres et les eaux qui sont sous le firmament des eaux qui sont au-dessus. Puis, au milieu de l'espace ainsi délimité, il crée la terre habitée. Ce serait donc un « lieu » ayant une substance, placé au milieu d'un « lieu » qui est l'univers organisé grâce aux « limites ». On comprend mieux pourquoi Éphrem n'admet pas un lieu incorporel.

Quelle pourrait être la position stö̈cienne qu'il combat ? Il s'agit probablement toujours de la conception stoïcienne de la $\chi \omega ́ \rho \alpha$, mais dans son rapport avec le $\kappa \varepsilon v o ́ v$, en tant qu'étendue infinie de l'univers avec le vide autour ( $\tau$ ò $\pi \hat{\alpha} v$ ) qui contient le monde entier ( $\tau$ ò ő $\lambda o v)$, pour définir ce lieu qui renferme tous les corps. Or, cette conception du lieu a une portée théologique singulière dans un contexte doctrinal selon lequel le monde est $\mathrm{Dieu}^{52}$.

Étant donné que dans le fragment 9, Éphrem parle du lieu en des termes bibliques, il n'est pas impossible qu'il fasse allusion à l'idée stö̈cienne de

51. Voir Thesaurus syriacus, col. 3299-3310. On peut penser aussi au mot $\pi \varepsilon ́ p \alpha \varsigma$, même s’il ne figure pas parmi les traductions usitées.

52. À ce propos, Cicéron cite Chrysippe (De natura deorum, I, 39) et Sextus cite Zénon (M. IX, 104). Ce développement théologique de la physique stoïcienne a été exploité par Simplicius qui explique le nom de la déesse syrienne Atargatis comme « place des dieux » ( ó $\pi$ ov $\theta \varepsilon \hat{\omega} v$ ) ; voir In Ph. 641, 33. Sur le dieu-monde, voir Diogène Laërce, VII, 147-148. Pour la critique de cette doctrine : Plutarque, Sur les contradictions des stö̈ciens, 1051d11-1052b8; Sur les notions communes, 1074d12-1075e1 et 1085b6-c7 ; Sextus Empiricus, M. IX, 104. Sur Dieu immanent : Diogène Laërce VII, 135-138 ; Cicéron, De la nature des dieux, II, 22. Voir Pépin 1964 p. 131-135. M. Spanneut, dans son étude du stoïcisme chez les Pères de l'Église, note en conclusion que dans les théories sur Dieu, l'influence de cette philosophie est la moins importante ; voir Spanneut 1957 p. 426. 
dieu-monde, souvent confrontée à la conception biblique de Dieu présent « en tout lieu ». Le premier à le faire est Philon d'Alexandrie, lorsqu'il interprète l'expression « un certain lieu » dans le verset de Genèse 28, 11 sur le songe de Jacob ${ }^{53}$. Cette idée se retrouve chez les chrétiens qui eux aussi font appel comme Philon à l'idée d'un lieu incorporel pour dire que Dieu n'habite pas un lieu physique ; ils disent donc que Dieu « emplit tout », « contient tout », qu'il est « lieu de toute chose » ou encore qu' il est « son propre lieu $\gg^{54}$. Certes, les chrétiens n'iront jamais jusqu’à l'adhésion au panthéisme stoïcien. Cependant, ils ne dédaignent pas une certaine inspiration stoïcienne dans la doctrine de Dieu qui remplit et contient le monde.

Comparé à ses confrères, Bardesane est allé plus loin dans l'élaboration d'une doctrine cosmogonique d'inspiration stoïcienne. Du fait de la destruction de parties importantes du Discours contre le «De Domnus », nous ne pouvons pas savoir s'il avait abordé la conception théologique du dieu-monde. Cependant, elle semble avoir exercé une grande influence sur sa théologie. Quant à sa conception du dieu-lieu, Éphrem dit dans le Discours à Hypatios que « les louanges que Bardesane a prononcées au sujet du lieu sont plus grandes que celles qu' il adresse à Dieu » et qu'il traite le lieu comme une

53. « Mais il reste à examiner la troisième question qui va avec : qu'est-ce que le « lieu » qu'il rencontre ? Car il est dit : Il rencontra un lieu (Gen. 28, 11). Or le mot lieu ( peut avoir trois sens : premièrement celui de la place occupée par un corps. Il peut, dans une seconde application, désigner la Parole divine que Dieu lui-même a complètement remplie de Puissances incorporelles. Mö̈se dit, en effet : "ils virent le lieu où se tenait le Dieu d'Israël” (Ex 24, 10), le seul lieu où il leur est permis de sacrifier, leur interdisant ailleurs. [...] Troisièmement, c'est Dieu lui-même qui est appelé lieu, du fait qu'il contient toutes choses et n'est contenu absolument par rien, qu'il est le refuge de toutes choses parce qu'il est son propre lieu, étant contenu en lui-même et enveloppé par lui seul. » Philon d'Alexandrie, De Somniis I 61-63 (voir Savinel (trad.) 1962, p. 48-51). Voir aussi Sambursky 1977.

54. Les passages les plus importants : « [...] Et s'il ne se situe ni dans un autre monde, puisque Dieu emplit tout, ni autour d'un autre monde, puisque Dieu contient tout, c'est qu'il n'existe pas, puisqu' il n'y a pas de lieu où il soit », Athénagore, Supplique au sujet des chrétiens, VIII. « Or, c'est le propre du Dieu très haut et tout-puissant, et vraiment Dieu, d'être partout - bien plus, de tout surveiller et tout entendre - et de ne pas être contenu dans un lieu! Sinon, le lieu contenant serait plus grand que lui : le contenant plus grand que le contenu ; Dieu n'est donc pas contenu mais c'est le lieu de toute chose », Théophile d'Antioche, À Autolycus II, 3. $\ll[\ldots]$ On aura comme dieu le lieu, lui qui non seulement est plus grand que ton dieu, mais également est inengendré, incréé, et par là éternel et égal à ton dieu [...] », Tertullien, Contre Marcion, I, 15. Voir aussi : Clément d'Alexandrie, Stromata, VII, 5, 28 ; Origène, Traité des principes, I, 6. Sur cette idée chez les auteurs chrétiens, voir Spanneut 1957, p. 356. La question n'a rien perdu de son intérêt à l'époque d'Éphrem, car Grégoire de Nazianze lui consacre un long passage du Discours, 28, rédigé contre les disciples de l'arien Eunome : «Puisque pour nous la divinité est incorporelle, continuons notre enquête. N'est-elle en aucun lieu, ou en un lieu ?... Car si ce qui n'existe pas n'est en aucun lieu, ce qui n'est en aucun lieu n'existe probablement pas non plus. Et si elle est dans un lieu - puisqu'elle existe - elle est sûrement soit dans le tout, soit au-dessus de tout. »Discours 28, 10. 
idole par rapport à Dieu ${ }^{55}$. De manière générale, Éphrem accuse Bardesane de faire de Dieu un des éléments qui composent l'univers. Telle est sans doute la question fondamentale qui oppose ces deux auteurs sur le plan théologique. Bardesane pense le monde créé à partir des ìtyē ou estuksē, parmi lesquels figure le Dieu Créateur nommé « Grand $\bar{I} t y \bar{a} »$ ou « Seigneur des ìtyē ». Pour Éphrem, cette manière d'appeler Dieu porte atteinte à sa transcendance par rapport au monde créé ${ }^{56}$. Cette finalité théologique de la polémique contre Bardesane expliquerait alors pourquoi Éphrem discute avec tant d'ardeur la théorie des incorporels qui, à ses yeux, fait partie des concepts philosophiques qui fondent la doctrine théologique de son adversaire.

\title{
6. Le lieu comme un incorporel (fr. 10-12) : dimensions et limites
}

\author{
Fr. $10(14,16-14,31)$; Beck, fr. X $\left(13,47-15,13^{57}\right)$ \\ ... on a trouvé que deux choses sont vraies : il existe et il n'existe pas ; à savoir \\ qu'il existe en nom et en signification, <mais> qu'il n'existe pas en corps et \\ en substance. \\ Ce qui n'existe pas en substance n'a pas les trois dimensions (tlātā qyāminn) \\ non plus. Toute chose qui est substance ou corps, possède les trois dimen- \\ sions.
}

Fr. $11(16,23-16,42)$; Beck, fr. XI $(15,14-18,10)$

Sache donc que les bardesanites n'ont pas même entendu dire que les philosophes ... et voici, Bardesane a placé cette longueur et cette largeur dans les limites ${ }^{58} \mathrm{du}$ lieu, lorsqu'il dit que « de même ce lieu est délimité (etmšeh) par les choses qu'il contient $\gg^{59}$. S'il suppose que le lieu est <propre à être> limité (metšmeh̆), il est nécessaire que la longueur et la largeur appartiennent au lieu ; ceci est une position que j'ai contestée en parlant à ce propos.

55. Voir Discours à Hypatios, dans Mitchell (éd.) 1912, 133, 1ss. et 27ss. ; Beck 1976, p. 37-38, n. 42.

56. « Si un seul Dieu est proclamé, il proclame un seul “être”; parce qu'il n'y a qu'un seul Dieu, et l'être est aussi un seul. Il n'y a qu' un dans les deux, (comme) être et (comme) Dieu; et tout est saisi par un seul nom. Aucun autre Dieu n'est placé [à côté de Dieu], c'est une bêtise que d'en mettre par essence cinq à côté de lui », Éphrem, Contra Haereses, 3, 5 (Beck (éd.) 1957, vol. 1, p. 11-12); « Moïse témoigne [pour] nous qu’aucun autre n’a été appelé par le nom de l'Être ; ils sont nommés "dieux" et non "êtres"; sous un nom il enseigne le goût de sa bonté et sous l'autre nom il fait savoir la puissance de son être, afin qu'on puisse connaitre les deux. Il a révélé le nom à Moïse, il s'est appelé « Je suis » par lui-même; c'est le nom de l'être ; jamais il n'avait appelé par ce nom quelqu'un d'autre. Parce qu'il en appelle beaucoup par leurs noms, il a réservé un [seul] nom, afin qu'on puisse connaître qu'il est le seul être et aucun autre » (Contra Haereses, 53, 11-12 Beck (éd.) 1957, vol. 2 p. 204).

57. Beck prend en compte seulement une partie de ce texte, à partir de 14, 16.

58. Singulier dans le texte (mašwhteh d-atrā).

59. Beck trouve cette phrase - qui dit littéralement : « ce lieu est délimité par lui qui retient une quantité de choses »- peu claire et contradictoire par rapport à la doctrine stoïcienne du lieu corporel, à laquelle Bardesane semble adhérer ailleurs, notamment dans les passages concernant l'identification du lieu avec Dieu ; voir Beck 1976 p. 37-38, n. 42. 
Fr. $12(16,43-17,45)$; Beck, fr. XI $\left(15,14-18,10^{60}\right)$

... ainsi, ce qui <est $>$ longueur et largeur, pour toi les philosophes l'ont appelé ici incorporel. Et comme le lieu est limité (metmš́h $)$ en lui-<même $>$ et à lui-<même $>$, de même celles-ci (i.e. longueur et largeur) sont à prendre en considération en elles-mêmes et < par rapport> à elles-mêmes; de telle sorte que tu saches que ces choses sont séparées du lieu. Cela veut dire qu'elles sont des noms et des représentations mentales (šmāhè $w$-sukkālē), mais pas des corps et des substances (gušmè w-qnōmēe).

Tu délimites le corps qui possède la longueur. Et cette longueur, qui est née par le nom et appelée « longueur », tu l'as délimitée, mais tu ne peux pas lui donner les trois dimensions (tlātā qyāminn). Cette longueur est née entre le corps et la langue (melltāa). Ainsi le corps a fait naître la limite et la langue, ce nom qui dit «longueur ». Maintenant tu peux délimiter l'extension et la largeur de ce corps et quand tu l'as délimitée, tu appelles « longueur » ce que tu as délimité. Mais tu ne peux pas mesurer et délimiter le nom par lequel tu appelles « longueur », parce qu'il n'a pas de substance du tout. Le nom simple (litt. «nu ») est compris par le moyen de la signification (sukkālā) de la chose que tu délimites ${ }^{61}$.

Avec le fragment 10 on revient à la définition du corps comme possédant trois dimensions : «Et tout ce qui n'existe pas en substance, n'a pas trois dimensions (tlātā qyāmin) non plus. » L'expression syriaque tlātā qyāminn a

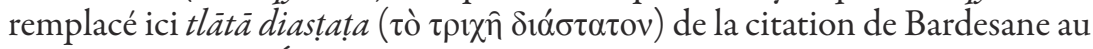
fragment 4. Ainsi Éphrem s'apprête-t-il à expliquer à ses lecteurs pourquoi les trois dimensions sont inséparables de la notion de substance.

Les fragments 11 et 12 concernent les dimensions et les limites du lieu, bien qu'Éphrem évoque seulement deux dimensions qui, techniquement,

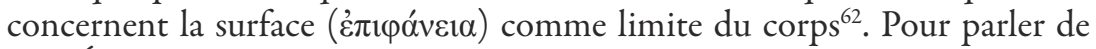
lieu, Éphrem utilise les termes provenant de la racine $m s ̌ h$, , qui possède deux sens : « oindre, marquer » (qui est à écarter) et « mesurer, limiter ${ }^{63}$. Nous choisissons de traduire « limiter / délimiter », parce que le débat porte sur l'extension du lieu et des choses. Le point de départ de cette polémique est une phrase de Bardesane, citée dans le fragment 11, qui dit : « ce lieu est délimité (etmšeh ) par les choses qu'il contient » - ce qui est une autre façon de dire que « un lieu est ce qui est occupé par un être et coextensif à celui

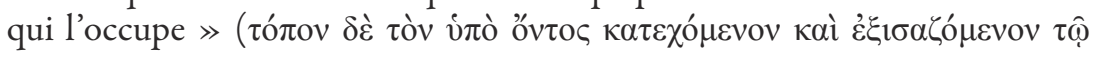

60. Le début de ce fragment n'est pas traduit par Beck. Il en traduit seulement la dernière phrase, en raison de lacunes mineures (16, 43-17, 5 ; voir n. 43).

61. Bevan \& Burkitt (éd.) 1921, 17, 11-45 ; voir Beck 1976 p. 38. La distinction entre la

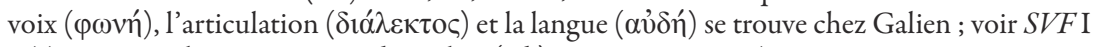
144. Voir aussi le commentaire de Hülser (éd.) 1987-1988 p. 524-527.

62. Voir Diogène Laërce, VII, 135 (SVF III, 259) ; Plutarque, Sur les notions communes, 1080d7-1081c2.

63. Thesaurus syriacus, col. 2235-2238. 


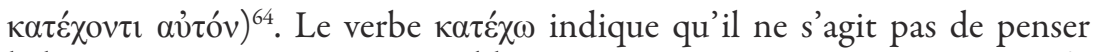
le lieu comme un espace mesurable, mais comme un espace occupé jusqu'à ses limites, voire délimité par l'être qui lui est coextensif. Revenir sur cette définition du lieu comme un continuum tridimensionnel, identique au corps qui l'occupe, permet à Éphrem d'aborder les notions de délimitations (longueur, largeur, profondeur).

Cependant, il semble que le long fragment 12 résume d'abord les positions stoïciennes concernant le caractère incorporel des notions telles que longueur et largeur, en tant que « limites de lieu » (mašwhtā $d$-atrā $)$. Éphrem précise que les « philosophes » ont appelé les dimensions du lieu « incorporelles », en les prenant en considération en elles-mêmes et non pas en tant que relatives au lieu. Cette position du problème ressemble beaucoup à ce que l'on trouve dans l'écrit du Pseudo-Galien, Que les qualités sont incorporelles, qui traite des trois dimensions en tant que qualités ( $\pi 010$ $\tau \eta \tau \varepsilon \varsigma)$ à travers lesquelles le lieu est défini et pensé ${ }^{65}$. Néanmoins, si Éphrem se réfère aux thèses stoïciennes, il le fait pour en tirer des conclusions différentes de celles du Pseudo-Galien (et de Sextus), puisqu'il ne traite pas les délimitations comme des qualités du lieu, mais comme des définitions. Il reconnaît leur existence en tant que « noms et représentations mentales ». En tant que telles, les dimensions sont bien des incorporels parce qu'elles ne sont pas « des corps et des substances ».

Arrivé à ce point, Éphrem étudie l'exemple de la longueur, en partant $\mathrm{du} \ll$ corps qui possède la longueur ${ }^{66}$. Cependant, lorsque l'on prend la longueur du corps en elle-même, il est impossible d'y retrouver les trois dimensions. La longueur, dit Éphrem, est née entre le corps, qui est long, et la langue qui connaît le mot «longueur ». Tout porte à croire qu'Éphrem se prépare à reconnaître le caractère incorporel des dimensions. Pourtant, ce n'est pas ce qu'il fait. En revanche, il reproduit ici son raisonnement précédent : il est possible de délimiter un corps, en lui attribuant les trois dimensions ; mais les mots «longueur », « largeur » et « profondeur » n'ont pas de substance, ils ne viennent pas du corps en tant que tel mais du fait de

64. Il s'agit de l'opinion rapportée par Sextus Empiricus (voir SVF II, 163). On peut la

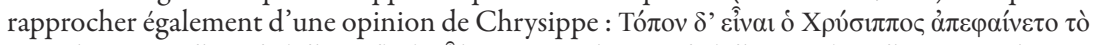

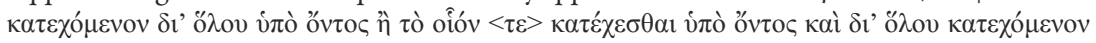

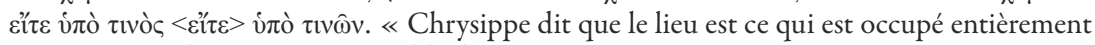
par un être, ou bien ce qui est capable d'être occupé par un être et qui est occupé entièrement soit par quelque être, soit par quelques êtres » (SVF II, 162). Sur le lieu comme limité par les extrémités et par le vide, voir Inwood 1991.

65. Pseudo-Galien, Que les qualités sont incorporelles, 2-8; voir Giusta (éd.) 1976 p. $27-29$. L'autre référence est Sextus Empiricus, P. III, 124-127.

66. « La surface est la limite d'un corps, ou bien ce qui a seulement une longueur et une largeur, mais n'a pas de profondeur... La ligne est la limite d'une surface ou une longueur sans largeur ou encore ce qui a seulement une longueur. Le point est la limite de la ligne, ce qui est la marque minimale », Diogène Laërce VII, 135 (SVF III, 259). Voir aussi Bréhier 1997 [1907] p. 41-42. Sur le vocabulaire éphremien, voir Possekel 1999 p. 165-166. 
pouvoir saisir la « signification », sukkālā, du nom par lequel ce corps est désigné ${ }^{67}$.

Le fragment 12 est une très bonne illustration de la manière dont Éphrem modifie la théorie stoïcienne des incorporels, notamment dans sa partie concernant les distinctions entre le nom et la représentation mentale. Le lien entre le corps et le nom, annoncé ici, est essentiel pour comprendre la nature de cette modification, dont Éphrem se servira encore. En ce sens, le fragment 12 se révèle important pour la suite du texte. Éphrem étudie, pas à pas, les composantes de la définition de l'incorporel pour la formuler ainsi : l'incorporel, premièrement, est conçu par la pensée, deuxièmement, ne possède pas de substance et, troisièmement, ne possède pas trois dimensions. Or, il semble que seuls certains « mots » correspondent aux exigences de cette définition; il faut alors préciser quelle est la conception éphremienne du langage.

\section{Nom, image, ligne - exemples des incorporels (fr. 13-15)}

\section{Fr. 13 (18, 25-43) ; Beck, fr. XII (18, 11-43)}

Je dis alors «j'achète » et « je vends »; la chose que j'achète ou que je vends est une certaine substance (qnōmā), mais ces mots du langage (mellē) et ces noms $(\stackrel{s}{s} m \bar{a} h \bar{e})$ qui se prononcent « achat » et « vente » n'ont pas de substance propre. Cependant, les substances qui sont achetées ou vendues ont ces trois dimensions (tlätā qyämìn). Or, ces noms ne l'ont pas, car ils sont incorporels.

Fr. $14(18,44-19,17)$; Beck, fr. XIII $(18,44-19,17)$

Il est inutile de t'écrire beaucoup au sujet du temps et du nombre, car tu en as déjà entendu < parler >, ainsi qu'au sujet de tout ce qui est incorporel ; tout cela est comme ces choses ou leur ressemble. Ses branches s'étendent <à partir d'>ici, de leur racine, celle-là même à partir de laquelle poussent toutes les branches. Il aurait suffi qu'au moyen d'une seule tu <les $>$ connaisses tous, mais eux (i.e. les philosophes) ont abondamment montré ces choses à celui qui les cherche, afin de parer à la faiblesse de celui qui questionne.

Fr. 15 (19, 18-20, 44); Beck, fr. XIV (19, 18-20, 44)

Écoute encore au sujet du point (smīyon) et de la ligne (surțāa) ; ce sont aussi des incorporels relatifs aux substances solides, à savoir le cheval ou l'aigle ou l'un des <êtres> corporels et des substantiels ... tu sais que si quelqu'un veut peindre (sar) le cheval ou le lion, avant que le peintre ne peigne sur la tablette, l'image du cheval est peinte dans son esprit. Et si le peintre veut ajouter un membre <en plus >, tu le blâmes à cause de la substance du cheval qui est la contrainte réelle.

67. La dernière phrase du fragment 12 est à rapprocher du passage de Sextus Empiricus sur

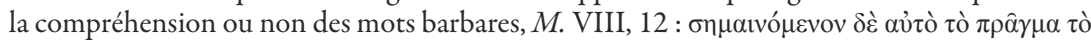

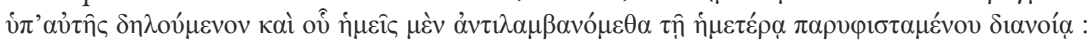
« la chose signifiée est alors une chose réelle indiquée par celle-ci et que nous appréhendons comme existante en dépendance de notre intellect.» 
Mais si je te dis que j'ai l'intention de tracer une ligne (surțā), tu ne sais pas quelle <ligne > je tracerai pour toi, car une ligne n'a pas de substance, comme la substance que possède un cheval et tu le (i.e. peintre) blâmes quand il $\langle y\rangle$ ajoute ou soustrait. Mais si tu penses : « il a tracé une ligne droite », il a tracé une courbe ; et si tu penses qu'il fait un quadrilatère, il a pu faire un octogone. Quand le peintre peint les images des corps (șîrin șìrā dmütē $d$-gušmēe) qu' il perçoit, il ne peut rien leur ajouter ou leur soustraire. Et quand il peint l'image des substances (dmūtā $d$-qnōmēe qu'il ne peut pas percevoir, il les peint au moyen de leurs couleurs et de leurs formes $(d m \bar{u} t \bar{e})$. Et si, à l'une des substances, il ajoute quelque chose qui n'appartient pas à la <vraie> image de cette substance (yuqāneh d-qnōmā), il est blâmé. Dans le cas d'une ligne, il ajoute et soustrait tout ce qu'il veut, et il n'est pas blâmé ; parce que l'image de la ligne ne possède pas de vraie substance, tu ne peux pas le blâmer. Et parce qu'elle (i.e. la ligne) n'a pas de substance, elle n'existe pas, et parce qu'elle n'existe pas, pour cette raison elle est aussi appelée incorporelle.

Les trois fragments parlent de différents types d'incorporels et l'on se rend vite compte que les exemples éphremiens ne sont pas les mêmes que ceux dont parlent les stoïciens. L'exemple cité dans le fragment 13 est hautement problématique. Par la distinction entre un objet acheté et le nom de l'action « j'achète », Éphrem supprime un troisième élément, le signifié. Son propos est d'autant plus inattendu que dans le fragment 12, il a bien présenté la « longueur » en tant que signifiée, distincte du corps qui est long et de la langue qui y met un nom, et il a même introduit le terme sukkālà. La seule explication de cet exemple, qui paraît incongru et éloigné des concepts stoïciens, serait qu'Éphrem se prépare à remplacer la théorie stoïcienne par sa propre conception du rapport entre le nom et la chose, dans lequel le signifié ne joue pas le même rôle, ou bien n'en joue aucun. En prétendant que les mots du langage (mellè, sg. melltā) et les « noms » (šmāhēe) sont aussi des incorporels, Éphrem abandonne définitivement la conception stoïcienne (et corporelle) de la langue, avec ses distinctions complexes concernant le discours, le nom, la langue, le son et l'exprimable incorporel ${ }^{68}$. Il reviendra encore sur cette question plus loin.

Dans le fragment 14, Éphrem règle, en quelques mots, la question du temps et du nombre (il les mentionne encore dans le fragment 16) ${ }^{69}$; il les déclare simplement incorporels, en se limitant à dire qu'il le fait sur la base des mêmes critères que ceux en rapport avec le lieu, déjà exposés.

68. Le raisonnement d'Éphrem serait à rapprocher de celui de Clément d'Alexandrie dans Stromata, VIII, 9, 26, 4-5, dans lequel il distingue seulement entre la « maison » incorporelle, en tant que nom, et la « maison » corporelle; voir Atherton 1993 p. 284-285. Voir aussi Long 1971 et Brunschwig 1995.

69. Éphrem n'aborde pas les notions de temps et de nombre séparément. Pour les stoïciens, le temps est incorporel (Sextus Empiricus M. VII, 104 ; X, 218, 227 ; Diogène Laërce, VII, 141). Le nombre l'est pour les pythagoriciens (Sextus Empiricus M. IX, $364 ; P$. III, 152). 
Le fragment 15 commence par la mention du point ( $\sigma \eta \mu \varepsilon i ̂ v)$ et de la ligne $\left.(\gamma \rho \alpha \mu \mu)^{\prime}\right)$, mais la réflexion qui suit ne porte ni sur les figures géométriques ni sur leurs délimitations ${ }^{70}$. Autant que le mauvais état de conservation du texte permette d'en juger, le fragment 15 donne une interprétation singulière de la ligne (surțā), comparée avec l'image (dmūta $)$. Le passage de l'une à l'autre se fait à l'occasion d'une description détaillée de l'activité du peintre (șì̄a). Éphrem réalise ainsi une comparaison inédite, conditionnée par une conception de l'image en tant qu'imitation d'une chose sensible. Il montre alors les différences entre les figures géométriques qui correspondent aux objets conçus dans la pensée et les peintures qui imitent les objets sensibles. Le but d'Éphrem est de montrer comment la ligne n'est pas l'image d'une substance, ce qui lui permet de dire qu'elle est privée de substance propre et que, pour cette raison, elle est incorporelle. De fait, les figures géométriques n'imitent aucune substance. À la différence de la ligne, l'image a pour but de ressembler à une substance. C'est même le critère permettant de juger la qualité de l'image : elle doit représenter les corps, sans qu'un peintre puisse leur soustraire ou ajouter quoi que ce soit. On pourrait dire que l'essentiel de l'image est dans le corps ou la substance représentée. Mais Éphrem n'oublie pas que la ligne fait, elle aussi, partie de l'image. Dans le fragment 18, il dit : «Il n'y a rien qui soit peint ou écrit, sans qu'il ait une ligne (surțā) dans sa peinture (b-surteb) ou dans son écriture (b-ktabeb). » La ligne (surțā) n'est pas uniquement une figure géométrique, puisqu'elle fait aussi partie de la peinture ou de l'écriture.

La manière dont Éphrem définit l'image comme imitation des substances nécessite quelques mots de commentaire. Le terme utilisé en syriaque pour image est $d m \bar{u} t \bar{a}$, dont la caractéristique essentielle est la ressemblance avec la chose représentée. La ressemblance est fondamentale : c'est elle qui donne un « corps » aux traces de la peinture sur une tablette, permettant d'identifier la substance de ce qui est représenté. La ressemblance avec la substance est la définition même de l'image ; d'ailleurs, tel est le sens du verbe dma: « ressembler », « imiter ». La valeur de cette image réside dans sa valeur mimétique $^{71}$. Pour souligner la réalité de l'image, Éphrem utilise yukānā, qui n'est rien d'autre que le terme grec દíẃv transcrit en syriaque. On le traduit par « vraie image », pour souligner que cette image réunit deux types d'imitation, celle de la substance et celle de l'idée de l'objet représenté. Sur ce point, le mot syriaque qnōmā, habituellement traduit par « substance »,

70. Cf. le commentaire aux fragments 11 et 12 dans la section précédente sur les limites. U. Possekel pense qu'Éphrem partage une opinion commune au sujet de l'incorporéité des objets géométriques (Sextus Empiricus M. III, 22-37 - voir Possekel 1999 p.165-169).

71. Dans ce rapport à la substance, il y a quelque chose d'assez proche de l'esthétique stoïcienne, notamment vis-à-vis de son rapport à la nature et de sa valeur mimétique - voir Zagdoun 2000 p. 69-78 et p. 147-160. 
est plus proche de « chose réelle » ou « chose existante en elle-même ». Le peintre doit d'abord former, dans son esprit, une certaine image de la chose à peindre et ensuite y conformer son art. Il s'agit donc de transformer ce qui est tracé, comme ligne sans substance, en image « attachée » à une substance concrète, sans qu'on puisse lui « ajouter ou soustraire » quelque chose, car si cette substance de référence est susceptible de croître ou de diminuer, la chose n'a pas de substance, n'existe pas et est incorporelle ${ }^{72}$.

\section{Le nom et l'incorporel (fr. 16-18)}

Fr. $16(22,23-23,37)$; Beck, fr. XVI $(22,23-36)$ et fr. XVII $(22,37-24,20)$ Je te dis alors brièvement, il y a ces trois groupes d'incorporels : un groupe de noms fixes (šmāhè asìrē) donnés aux corps et aux substances, un <deuxième groupe > de noms donnés aux significations (sukkālē), comme celles de lieu et de temps et de nombre et un autre groupe de mots (mellē) prononcés au sujet de tout $<$ le reste $>$. Quoique ces trois groupes soient des incorporels, il y a seulement sept noms <qui > désignent des incorporels. Et pourquoi ceux-là <seulement>, et non les noms 《 or » et « argent » ? Même si ces noms-ci sont des asomata (asōmata), c'est-à-dire des incorporels, cependant, parce qu'ils ont été donnés à des corps et à des substances, ce sont aussi des noms corporels (šmāhè gršmēè). Ainsi quand tu entends un nom que quelqu'un a dit et prononcé « or » ou « argent » ou « aigle » ou « terre », à la simple mention du nom, ton imagination se fixe sur la substance corporelle (qnōmā gšima). Et tu sais si cela est dur ou mou, ou doux ou amer. Et de même quand on te parle de couleurs. Par contre, si on te parle du temps ou du nombre, ton imagination ne se fixe pas sur les corps ou les substances. Quelle est la substance du temps ? Ou quel est le corps du nombre ou du lieu ? Et tu ne sais pas s'il est noir ou blanc, ou doux ou dur.

Fr. 17 (24, 1-48); Beck, fr. XVII (22, 37-24, 20)

Quelqu'un a dit « longueur », sans avoir précédemment <prononcé> « corps » et ainsi ses extensions ne sont pas exprimées <par> le nom longueur. « La longueur et la largeur, voici des noms qui existent par euxmêmes, sans le support du corps. » Et après, il a dit : « La longueur et la largeur d'une pierre, voici, elles habitent les corps comme des qualités. » Mais si tu dis «fer » ou « cuivre », avec son nom ... aussi la substance. Et le nom de fer ou de cuivre ne peut pas dire substance tout (?) ... Et pour cela leurs noms sont fixés (asīiē). Ces choses ... sont fixées sur les substances, ne sont pas unies avec les noms de fer ou de cuivre ou de pierre ... Et il convient (?) que le nom < qui est> incorporel soit détaché (śriyā) d'eux, parce qu'ils ne sont pas comme fixés sur la substance, peut-être à cause de cela sont-ils appelés asomata (asōmata). »

72. On se demande dans quelle mesure cette idée dépend de l'Argument Croissant ( $\alpha \mathfrak{c} \xi \alpha v o ́ \mu \varepsilon v o \varsigma \lambda{ }^{\prime} \gamma \circ \varsigma$ ), un paradoxe auquel s'oppose la notion stoïcienne d'identité. Plutarque (Sur les notions communes, 1083b-c) est une des sources principales pour cette question - voir Sedley 1982 et Brunschwig 1995 p. 136-139. 
Fr. $18(26,2-26,41)$; Beck, fr. XVIII $(26,2-26,41)$

Et encore un autre type <d'argument $>$. Il n'y a rien qui ne soit pas nommé comme étant dans un lieu (atrā) ou dans un espace $(d u k t \bar{a})$. Il n'y a rien qui ne soit dans le temps, soumis au temps et effectué dans le temps. Il n'y a rien qui ne soit pas ou qui ne devienne pas l'objet du nombre et de la mesure. Il n'y a rien qui ne possède la longueur, la largeur et l'étendue. Il n'existe aucun corps ou personne (parșopā) qui n'ait pas de signe ( $\bar{a} t \bar{a})$ lui permettant de se distinguer de son compagnon, comme le point qui distingue un mot <écrit> d'un autre. Il n'y a rien qui soit peint ou écrit, sans qu'il ait une ligne (surțā) dans son dessin (b-surteh) ou dans son écriture (b-ktabeb). Il n'y a pas de bruit [d'instrument] ou de chant ${ }^{73}$, pas de murmure ou de voix sans qu'il y ait un de ces sept sons (qālēe, ou <un> de ces sept sons vocalisés (hegyānēe), ou une des sept syllabes (sullabiyê). Et pour cela, même ici les stoïciens ont conçu les sept syllabes incorporelles (sullabiyé asōmata), afin qu'on puisse inclure en elles toutes choses, comme ces lettres qui n'ont pas de voix (cf. $\alpha \varphi \omega$ $v \alpha \gamma \rho \alpha ́ \mu \mu \alpha \tau \alpha)$.

Les trois derniers fragments sont particulièrement difficiles à interpréter, parce qu'ils évoquent ce qui a été déjà dit au sujet de la langue. Pourtant, ils contiennent l'essentiel de la conception éphremienne de l'incorporel, préparée par les argumentations précédentes qui ont permis au lecteur d'en apercevoir déjà les grands traits. On constate que la théorie exposée dans le fragment 16 n'est pas reçue sans réserve et, dans le fragment 17, on trouve des marqueurs de citation. Le plus curieux est le fragment 18 qui contient des thèses proches du stoïcisme, mais employées pour montrer que l'incorporel n'existe pas. Il y a aussi une translittération des termes grecs, si caractéristique de l'écriture de Bardesane. On ne peut pas nier que ces textes transmettent les opinions d'Éphrem, parce qu'ils s'accordent avec ses vues déjà exposées sur l'incorporel, mais ils semblent montrer également que sa polémique avec Bardesane ne se laisse pas réduire à une adhésion opportuniste aux thèses platoniciennes d'Albinus.

Le fragment 16 donne une classification des noms. On a déjà rencontré dans les fragments 6, 12 et 13 la notion de « nom » $(\check{s} m \bar{a})$ qui apparaissait en filigrane. On pense donc à la définition de l'incorporel comme « ce qui existe en nom $\left(b-\right.$ šm $\left.^{2} \bar{a}\right)$ et en signification $(b$-sukkāla $) \gg$, dans les fragments 6 et 12 , ainsi qu'à la distinction entre « nom » $\left(s^{\prime} m \bar{a}\right)$ et « langage » (mellta $)$, dans le fragment 13. Dans le fragment 16 , les noms - qui sont dits incorporels - sont classés en trois groupes, selon leur rapport à la substance. Il y a des noms « fixés » sur la substance, des noms relatifs à la signification conçue seulement dans l'intellect (où l'on trouve les incorporels stoïciens) et des noms « au sujet de tout ». Cette classification et ses critères ne semblent

73. Éphrem utilise deux termes très proches nqāāā et $z m a \bar{a} m a \bar{a}$. Pour les distinguer, Beck se réferre aux autres écrits d'Éphrem, où le premier est relatif aux sons d'un instrument (d'une harpe) et le second au chant ; voir Beck 1976 p. $46-47$ n. 82 et 83. 
pas particulièrement stoïciens ${ }^{74}$. Éphrem résume correctement l'essentiel de cette doctrine, mais sans y adhérer totalement, semble-t-il. C'est le caractère « incorporel » de certains noms qui lui pose problème. Or, selon lui, même ces noms-là, en réalité, « sont des noms corporels (šmāhē gřšmēè) ». Sa critique s'appuie sur le fait qu'il supprime systématiquement le signifié, en distinguant seulement entre la chose et le nom. Cela reste une argumentation très surprenante, parce qu'il emploie sans difficulté le terme syriaque correspondant au signifié, à savoir sukkālā.

Le sukkālā, qui a déjà été utilisé dans les fragments 6 et 12, correspond

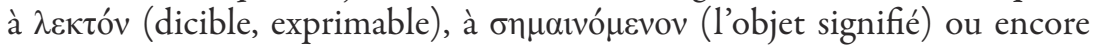
à $\pi \rho \hat{\alpha} \gamma \mu \alpha$ (élément logique ou unité du sens), dont les connotations sont multiples ${ }^{75}$. Pour rendre compte de son rôle dans l'argumentation d'Éphrem, nous avons choisi de le traduire par « signification », eu égard à une partie de la définition éphremienne de l'incorporel en tant que « ce qui est conçu par la pensée $\gg^{76}$. Notons cependant qu'Éphrem n'utilise pas le sukkālā pour désigner les objets imaginés par le peintre, parce que ceux-ci seraient de l'ordre de la $\varphi \alpha v \tau \alpha \sigma i ́ \alpha \alpha i \sigma \theta \eta \tau \imath n^{77}$. Dans le fragment 16, sukkālā figure dans la phrase concernant la catégorie des « noms qui sont donnés à des significations (sukkālēe), comme ceux de lieu, de temps et de nombre ». Il s'agit donc de réalités concevables indépendamment de la notion de substance. En comparaison avec la substance et la catégorie des « noms attachés à la substance », le sukkālā remplirait le même rôle vis-à-vis des « noms donnés aux significations » - c'est ainsi qu' il assure à ces noms leur statut de « détachés » de la substance.

L’explication à propos des noms « attachés » et « détachés » se trouve

74. Cette classification ne respecte pas la distinction entre le signifiant, le signifié et le porteur extérieur (voir $S V F$ II, 166), ni la théorie de l'origine naturelle des noms (voir SVF II, 146). Voir aussi Brunschwig 1995. Cette classification ne tient pas compte de la voix, qui est pourtant un élément essentiel dans la théorie stoïcienne du langage (voir SVF II, 136-165).

75. Diogène Laërce définit ainsi le terme $\pi \rho \hat{\gamma} \gamma \mu \alpha$ : « on exprime (par la parole) des

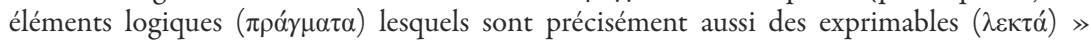
(DL VII, 57). Comme le montre P. Hadot, les $\pi \rho \alpha ́ \gamma \mu \alpha \tau \alpha$ peuvent être traduits de plusieurs manières : 1) ce dont on parle, 2) le sens, par opposition à ővo $\mu \alpha$ et $\lambda \varepsilon \varepsilon_{\xi}^{\prime}(\varsigma, 3)$ le concept par

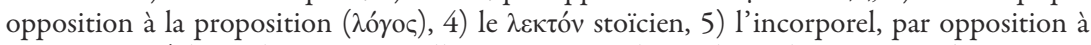

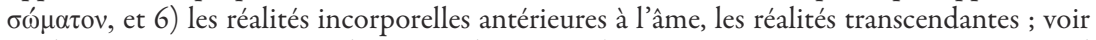
Hadot 1980. Compte tenu de ses nombreux emplois, on peut constater que sukkälā prend plutôt quelques-uns des sens de $\pi \rho \hat{\gamma} \gamma \mu \alpha$ indiqués par 2, 3, 4 et 5 . Hadot propose de traduire $\pi \rho \hat{\gamma} \gamma \mu \alpha$ par « unité de sens », ce qui convient bien à sukkālā utilisé par Éphrem.

76. Ce choix est fondé également sur la définition du $\lambda \varepsilon \kappa \tau$ óv incorporel en tant que ce qui « subsiste en conformité avec notre pensée », transmise par Sextus Empiricus dans M. VIII, 70 (SVF II, 187 ; SVF II, 166-171). Pour les références à Sextus à propos de l'expression «ce qui est conçu par la pensée », voir Beck 1976 p. 32 n. 25.

77. Cf. Bevan \& Burkitt (éd.) 1921, 20, 45-22, 22. Le fragment concernant les objets imaginés par le peintre correspond au fragment XV de Beck et il se trouve entre nos fragments 15 et 16. Sur la $\varphi \alpha v \tau \alpha \sigma i ́ \alpha$ des stoïciens, voir SVF II 83. 
au fragment 17 , très lacunaire, dans lequel on trouve également deux lam, marqueurs de citation. Les passages cités concernent les délimitations du corps tridimensionnel, considérées comme des qualités du corps. Éphrem objecte, en simplifiant la question : les qualités sont toujours relatives au corps et à sa substance. Une fois de plus, il confond le nom et le signifié, en distinguant seulement entre la substance et le nom. Ici, l'on se rend compte qu'Éphrem fait dépendre le statut de somata et asomata du lien entre le nom de l'être et la substance, voire entre le nom et le corps, et non du fait de savoir si l'être en question est lui-même un corps ou non.

Cette étrange compréhension de la notion d'《 incorporel » apparaît pleinement dans le fragment 18. Éphrem dresse une liste d'incorporels qu'il avait annoncée dans le fragment 16 , en parlant de « seulement sept noms des incorporels ». Ici, apparaît une liste sur laquelle figurent 1) le lieu, 2) le temps, 3) le nombre, 4) les extrémités : limite, longueur, largeur, 5) le point et signe, 6) la ligne, 7) le son ${ }^{78}$. Deux observations doivent être faites par rapport à cette liste. Premièrement, chaque catégorie d'incorporels est citée pour être contestée; il n'y a rien qui ne soit pas nommé par rapport à un lieu, un temps, qui ne soit pas mesurable ou dénombrable, ou qui soit privé d'un signe distinctif ou d'un point, d'une ligne ou d'un son. Cela peut vouloir dire que chaque être est, d'une certaine façon, un « incorporel » ou bien que chaque incorporel est, d'une certaine façon aussi, attaché à la substance. En remettant en question la possibilité d'utiliser le terme « incorporel », Éphrem tente de saper les fondements de la doctrine stoïcienne. Il n'est pas question pour lui de dire que l'incorporel n'existe pas : dans ce cas, il aurait adhéré à la doctrine stoïcienne selon laquelle tout ce qui existe est un corps. Le fait que son argumentation porte principalement sur des catégories linguistiques indique que c'est la notion même d'incorporel qui est l'objet de sa critique. Éphrem trouve cette notion superflue et contradictoire, en reprenant à son compte les critiques formulées par les platoniciens et, probablement, relayées par Albinus. Cependant, cette tentative paraît plutôt maladroite, parce que - et c'est notre deuxième observation - sur la liste des « incorporels », les points 1-4 correspondent aux incorporels stoïciens, auxquels s'ajoutent des êtres classés parfois comme corporels et parfois comme incorporels, comme le son qui est corps pour les stoïciens et incorporel pour les platoniciens ${ }^{79}$.

La fin du fragment 18 traite des composants de la « voix » : Éphrem dit que dans chaque être il y a un son $(q \bar{a} \bar{l} \bar{a})$ qui correspond soit à $\varphi \omega v \eta ́$, soit, moins souvent, à $\lambda \varepsilon^{\prime} \xi 1 c$. Il mentionne donc « sept sons $(q a \bar{l} \bar{e}) »$, « sept sons vocalisés $($ begyānē $) \gg$ et « sept syllabes (sullabiyē $) \gg$. Selon les stoïciens, la

78. L'analyse de la phrase du fragment 16 par rapport à la suite du texte est faite par Beck 1976 p. 46 et Possekel 1999 p. 172-173.

79. Voir Timée, 67b. La différence entre les platoniciens et les stö̈ciens sur ce point est exprimée par Aulu-Gelle, Noctes Atticae, V, 15, 1-7. Sur la voix, voir Mansfeld 2005. 


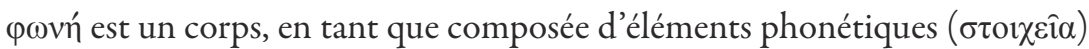
ayant les lettres $(\gamma \rho \alpha ́ \mu \mu \alpha \tau \alpha)$ pour « signes » ou « points ${ }^{80}$. Quant aux lettres, Diogène Laërce parle de sept lettres « vocales » ( voyelles $(\alpha, \varepsilon, \eta, 1, o, v, \omega)$ et six lettres « aphones » ("丷 $\varphi \omega v \alpha)$ ou muettes $(\beta, \gamma$, $\delta, \kappa, \pi, \tau)^{81}$. Les « lettres qui n'ont pas de voix » seraient donc des consonnes muettes ( $\alpha \alpha \omega v \alpha \gamma \rho \alpha ́ \mu \mu \alpha \tau \alpha)$. On constate qu'Éphrem dispose d'informations très précises, provenant sans doute de l'écrit de Bardesane, l'objet de sa critique. Cependant, on ne comprend toujours pas à quoi correspond l'expression « les sept syllabes incorporelles (sullabiyē asōmata) ». Elle fait penser à un système musical où les sept notes / sons fonctionnent à la façon

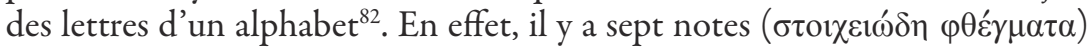
et dans les théories musicales, elles sont à la base d'un système qui part de la

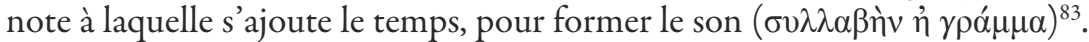
Éphrem serait alors en train de confondre deux systèmes, linguistique et musical, dans son effort pour montrer que la notion d'incorporels n'a aucune raison d'être utilisée car tout ce qui existe est, d'une façon ou d'une autre, lié à la substance, voire au corps.

\section{Conclusion}

Étant donné que le critère fondamental de la distinction éphremienne entre corporel et incorporel est le « lien » avec la substance sensible, il est

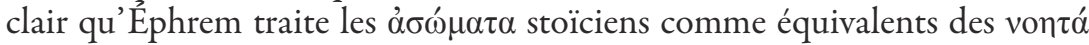
platoniciens ; mais, dans un deuxième temps, il fait appel au matérialisme stoïcien pour montrer que les von $\tau$ ó sont liés aux corps. Cette confusion vient en partie de l'écrit de Bardesane, qui est sa source d'information sur ce qui est « incorporel ». Ces notions ont été certainement utilisées par Bardesane et par Albinus, mais la lecture des fragments que nous avons rassemblés montre qu'Éphrem n'a pas saisi les nuances de la polémique. De toute évidence, le théologien possédait une certaine formation philosophique, mais très

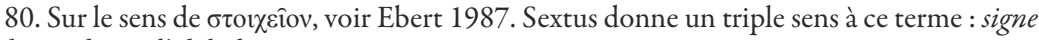
graphique, lettre d'alphabet, signe peint.

81. Diogène Laërce, VII, 57. Cependant, comme l'observe J.-B. Gourinat au sujet de cette liste, le terme « lettre » $(\gamma \rho \alpha ́ \mu \mu \alpha)$ possède un triple sens : l'élément phonique, le caractère écrit et son nom. Ainsi, les lettres manquantes, à savoir les semi-voyelles $(\zeta, \lambda, \mu, v, \xi, \rho, \sigma, \psi)$ qui ne peuvent pas former une syllabe et les muettes rauques $(\theta, \varphi, \chi)$ traitées parfois comme des semi-voyelles, auraient pu ne pas faire partie de la liste en raison de leur valeur phonique ; voir Gourinat 2000 p. 148-149.

82. C'est l'analyse faite dans Possekel 1999 p. 178-181. Elle se réfère aux écrits d'Aristide Quintilianus et de Plutarque. Beck opte quant à lui pour une interprétation linguistique, appuyée sur les écrits de Sextus Empiricus; voir Beck 1976 p. 45-47.

83. Voir Pseudo-Plutarque, De musica, 1144a8-10. Le passage est étudié par Possekel 1999 p. 180. Les stoïciens s'intéressent à la voix humaine en tant que son censé imiter le son des instruments de musique ; voir Gourinat 2000 p. 149-150. 
sommaire et fortement dépendante de la tradition doxographique qu'il a connue en syriaque plutôt qu'en grec ${ }^{84}$. Pourquoi a-t-il entrepris l'examen critique d'un débat dont il n'était pas familier?

Nous avons déjà montré quelques implications théologiques de la doctrine de l'incorporel, notamment pour tout ce qui concerne la présence de Dieu dans le monde. Après avoir parcouru tous les fragments relatifs à cette doctrine, on peut développer une explication. L'analyse de la liste des « sept incorporels » du fragment 18 faite par E. Beck montre en réalité que les quatre premiers au moins sont mentionnés ailleurs par Éphrem : dans les textes antiariens, le lieu, le temps et le nombre appartiennent aux choses créées par Dieu ${ }^{85}$. Autrement dit : toutes les choses créées existent dans un lieu et dans un temps; elles sont également dénombrables, délimitées, exprimables par la voix, imaginables par la pensée. Par toutes ces caractéristiques, les créatures s'opposent à leur Créateur qui seul n'est pas limité par le lieu, le temps, le nombre et le nom. Ainsi Éphrem semble-t-il défendre la position selon laquelle Dieu est le seul être « incorporel ». Vu de cette manière, le terme « incorporel » n'est pas pris au sens stoïcien. Malheureusement, cette position théologique n'est pas explicitement formulée dans la partie conservée du texte et notre seul passage qui témoigne d'un intérêt théologique porté à la notion d'incorporel se trouve à la fin de la partie du Discours dédiée à cette problématique. Il s'agit d'une curieuse interprétation de ce qu'est la surface :

Celui qui trace la ligne a dit au sujet de la géométrie (gematrūtāa que dans ces lignes sont réalisées toutes les créatures et toutes les œuvres. Et que les Grecs appellent la surface (epipanya) « révélation » (galyütā); elle est donc l'apparition de tout ce qui existe, car il n'y a rien de visible et d'invisible qui n'ait pas sa propre apparence ${ }^{86}$.

Le raisonnement d'Éphrem n'est pas évident, bien qu'il soit assez

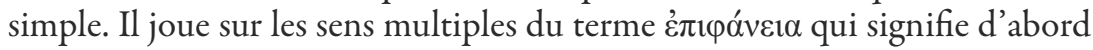
« l'apparition » et « la surface » mais qui, dans le langage chrétien, est aussi synonyme de révélation, terme théologique par excellence. Éphrem passe alors d'un sens à l'autre et considère la surface - au sens mathématique comme un lieu où les choses apparaissent en tant que créatures de Dieu,

84. Éphrem a sans doute acquis ses connaissances en philosophie grecque dans le cadre de l'éducation générale, ou paideia, comme l'attestent les historiens du $\mathrm{V}^{\mathrm{e}}$ siècle ; voir Théodoret de Cyr, HE IV, 26 ; Sozomène, $H E$ III, 16. Les recherches modernes confirment qu'Éphrem connaissait seulement le vocabulaire grec et l'utilisation qu' il en fait ne permet pas de constater qu'il ait connu cette langue ; voir Possekel 1999 p. $52-53$; Beck 1976 p. 68.

85. Voir Beck 1976 p. 45-47. Les analogies citées par Beck viennent des Hymnes sur la foi $(H F)$, un recueil polémique antiarien, composé de quatre-vingt-sept hymnes. On y trouve les mentions du lieu et de l'espace ( $H F$ 30, 4), du temps $(H F 26,2)$, du nombre $(H F 30,1)$ et des trois dimensions (HF 30, 3) comme œuvres de Dieu.

86. Bevan \& Burkitt (éd.) 1921 p. 31, 34-46 ; Beck 1976 p. 53-54. 
visibles et invisibles. Cela confirme notre hypothèse : Éphrem considère les incorporels - qu'ils soient postulés par les stoïciens ou par les platoniciens comme des « créatures » et, par conséquent, inséparablement attachés aux êtres contingents. On comprend mieux alors pourquoi, dans le fragment 18, Éphrem remet systématiquement en question chaque catégorie des êtres dits « incorporels », en les attachant systématiquement à la substance, sinon à la matière. Il ne veut pas dire qu'ils n'existent pas, mais qu'ils ne sont pas vraiment 《incorporels ». Cependant, les critères qu'il utilise - ce qui est conçu par l'intellect et qui existe en nom et en signification - ne sont pas stoïciens. De ce point de vue, cette argumentation reproduit la structure des autres moments de la polémique contre Bardesane, notamment le reproche d'attribuer à Dieu $(\bar{I} t y \bar{a})$ le même nom que celui donné aux éléments dont est composél'univers (ìtyēou estuksēe $)^{87}$. Éphrem semble craindre qu'en détachant une créature de la substance, en lui accordant le statut d' « incorporel », on prenne le risque de la propulser « au-delà de la substance », dans la sphère de la transcendance et, à terme, de la rendre égale à Dieu.

Ainsi la manière dont Éphrem définit les incorporels à partir de leur rapport à la substance révèle une ambition véritablement théologique, ce qui est difficile à percevoir à première vue et à partir d'un texte fortement endommagé. La reconstruction de la portée théologique de la critique éphremienne permet de regarder autrement les maladresses et les simplifications qu'il opère dans ses efforts pour rendre compte d'un débat métaphysique d'une grande complexité. 


\section{BIBLIOGRAPHY}

Algra, K. 1995 : Concepts of Space in Greek Thought, Leiden, 1995 (Philosophia antiqua, 65).

Atherton, C. 1993 : The Stoics on Ambiguity, Cambridge, 1993 (Cambridge Classical Studies).

Baltes, M. 1996 : « Idee (Ideenlehre) », dans T. Klauser, E. Dassmann \& al. (éd.), Reallexikon für Antike und Christentum, Bd. 17 (Lfg. 129/136), Stuttgart, 1996, p. 213-246.

Beaujeu, J. 1973 (éd.) : Apulée, Opuscules philosophiques : Du Dieu de Socrate, Platon et sa doctrine, De Mundo : Fragments, Paris, 1973 (Collection des universités de France. Série latine, 211).

BECK, E. 1957 (éd.) : Ephraem, Des heiligen Ephräm des Syrer Hymnen contra Haereses, 2 vol., Leuven, 1957 (Corpus Scriptorum Christianorum Orientalium 169/Syri 76 ; Corpus Scriptorum Christianorum Orientalium 170/Syri 77).

— 1976 : «Ephräms Rede gegen eine philosophische Schrift des Bardaisan : übersetzt und erklärt », Oriens Christianus, 60 (1976), p. 24-68.

- 1978 : «Bardaisan und seine Schule bei Ephräm », Le Muséon, 91 (1978), p. 271-333.

Bevan, A. A. \& F. C. Burkitt 1921 (éd.) : Ephraem, S. Ephraim's Prose Refutations of Mani, Marcion and Bardaisan, of which the greater part has been transcribed from the palimpsest B.M. add. 14623 and is now first published, vol. 2 : The Discours called 'Of Domnus' and six other writings, London, 1921.

BrÉHIER, É. 1955 : « Le mot Nontòv et la critique du stö̈cisme chez Sextus Empiricus », Études dephilosophie antique, Paris, 1955, p. 193-206 (Publications de la Faculté des lettres de Paris. Série Etudes et méthodes, 1).

- 1997 : La théorie des incorporels dans l’ancien stoïcisme, 9e éd., Paris, 1997 [1907] (Bibliothèque d'histoire de la philosophie).

Brisson, L. 1998 : Le Même et l'Autre dans la structure ontologique du Timée de Platon: un commentaire systématique du Timée de Platon, $3^{\mathrm{e}}$ éd. rev. et corr., pourvue de corrigenda, d'addenda, d'index rev. et d'une bibliogr. analytique nouv. mise à jour, Sankt Augustin, 1998 (International Plato Studies, 2).

BRUNSCHWIG, J. 1995 : «Remarques sur la théorie stoïcienne du nom propre », Études sur les philosophies hellénistiques : épicurisme, stoïcisme, scepticisme, Paris, 1995 (Épiméthée), p. 115-139.

Camplani, A. 1998 : « Rivisitando Bardesane : note sulle fonti siriache del bardesanismo e sulla sua collocazione storico-religiosa », Cristianesimo nella storia, 19 (1998), p. 519-596.

Chiesa, M. C. 1991 : « Le problème du langage intérieur chez les stoïciens », Revue internationale de philosophie, 45, 178/3 (1991), p. 301-321.

Des Places, É. 1973 (éd.) : Numénius, Fragments, Paris, 1973 (Collection des universités de France. Série grecque, 226).

Dillon, J. 1977 : The Middle Platonists 80 B.C. to A.D. 220, Ithaca, 1977.

- 1993 (trad.) : Alcinoos, The Handbook of Platonism = Didaskalikos, transl., introd., comm., Oxford, 1993 (Clarendon Later Ancient Philosophers).

Donini, P. 1988 : « La connaissance de Dieu et la hiérarchie divine chez Albinos », 
dans R. van den Broek, T. Baarda \& J. Mansfeld (éd.), Knowledge of God in the Graeco-Roman World, Leiden, 1988 (Études préliminaires aux religions orientales dans l'Empire romain, 112), p. 118-131.

Dörrie, H. \& M. Baltes, 1993 (éd.) : Der Platonismus in der Antike, vol. 3 : Der Platonismus im 2. und 3. Jahrhundert nach Christus : Bausteine 73-100: Text, Übersetzung, Kommentar, Stuttgart, 1993.

Drijvers, H. J. W. 1966 : Bardaisan of Edesse, [transl. into English by G. E. Van Baaren-Pape], Assen, 1966 (Studia semitica neerlandica, 6).

EBERT, T. 1987 : « The Origin of the Stoic Theory of Signs in Sextus Empiricus », Oxford Studies in Ancient Philosophy, 5 (1987), p. 83-126.

Festugière, A.-J. 1954: La révélation d'Hermès Trismégiste, t. IV : Le Dieu inconnu et la Gnose, Paris, 1954 (Études bibliques).

Furlani, G. 1937 : « Sur le stoïcisme de Bardesane d'Édesse », Archív orientální, 9/3 (1937), p. 347-352.

Gersh, S. 1986 : Middle Platonism and Neoplatonism : the Latin Tradition, vol. 1, Notre Dame, 1986 (Publications in Medieval studies, 23).

Göransson, T. 1995 : Albinus, Alcinous, Arius Didymus, Göteborg, 1995 (Studia Graeca et Latina Gothoburgensia, 61).

Giò, A. 2002 (éd.) : Filosofi medioplatonici del II secolo D. C. : testimonianze e frammenti: Gaio, Albino, Lucio, Nicostrato, Tauro, Severo, Arpocrazione, Napoli, 2002 (Elenchos, 36).

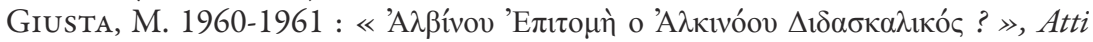
dell'Accademia delle Scienze di Torino, 95 (1960-1961), p. 167-194.

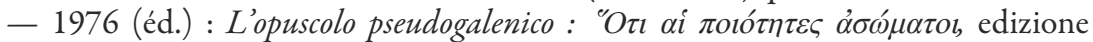
critica, traduzione e note, Torino, 1976 (Memorie dell'Accademia delle scienze di Torino. Classe di scienze morali, storiche e filologiche, Serie 4a, n. 34).

Gourinat, J.-B. 2000 : La Dialectique des stö̈ciens, Paris, 2000 (Histoire des doctrines de l'Antiquité classique, 22).

— 2013 : «Le discours intérieur de l'âme dans la philosophie stoïcienne », Chôra, 11 (2013), p. 11-22.

Hadot, P. 1980 : « Sur divers sens du mot pragma dans la tradition philosophique grecque », dans P. Aubenque (dir.), Concepts et catégories dans la pensée antique, Paris, 1980 (Bibliothèque d'histoire de la philosophie), p. 309-319.

Hugonnard-Roche, H. 1989 : « Aux origines de l'exégèse orientale de la logique d'Aristote : Sergius de Rešaina ( $†$ 536), médecin et philosophe », Journal Asiatique, 277/1-2 (1989), p. 1-17.

Hülser, K. 1987-1988 (éd.) : Die Fragmente zur Dialektik der Stoiker, neue Sammlung der Texte mit deutscher Übersetzung und Kommentaren, 4 vol., Stuttgart, 1987-1988.

InvERnIZZI, G. 1976 (éd.) : Albinus, Il Didaskalikos di Albino e il medio-platonismo, 2 vol., Roma, 1976.

Inwood, B. 1991 : «Chrysippus on extension and the void », Revue internationale de philosophie, 45 (1991), p. 245-266.

Jurasz, I. 2011-2012 : «Résurrection de l'âme chez Bardesane », Chôra, 9-10 (2011-2012), p. 399-427.

Le Corre, R. 1956 : «Le Prologue d'Albinus », Revue philosophique de la France et de l'Étranger, 146 (1956), p. 28-38. 
Long, A. A. 1971 : « Language and Thought in Stoicism », dans A.A. Long (éd.), Problems in Stoicism, London, 1971, p. 75-113.

Louis, P. 1945 (éd.) : Albinus, Épitomé, Paris/Rennes, 1945.

MANSFELD, J. 2005 : «'Illuminating what is thought' : a Middle Platonist placitum on 'Voice' in Context », Mmemosyne, $4^{\mathrm{e}}$ s. 58/3 (2005), p. 358-407.

Mazzarelli, C. 1980 : «L'autore del Didascalikos : l'Alcinoo dei manoscritti o il medioplatonico Albino ? », Rivista di Filosofia Neo-Scolastica, 72/4 (1980), p. 606-639.

Mitchell, C. W. 1912 (éd.) : Ephraem, S. Ephraim's Prose Refutations of Mani, Marcion and Bardaisan, of which the greater part has been transcribed from the palimpsest B.M. add. 14623 and is now first published, vol. 1 : The discourses addressed to Hypatius, London, 1912.

Moraux, P. 1984 : Der Aristotelismus bei den Griechen : von Andronikos bis Alexander von Aphrodisias. Band 2, Der Aristotelismus im I. und II. JH. N. CHR, Berlin, 1984 (Peripatoi, 6).

Orth, É. 1947 : «Les œuvres d'Albinos le Platonicien », L'Antiquité classique, 16/1 (1947), p. 113- 114.

- 1958: «Curae criticae », Emerita, 26 (1958), p. 201-213.

PÉPIN, J. 1964 : Théologie cosmique et théologie chrétienne (Ambroise, "Exam." I, 1, 1-4), Paris, 1964 (Bibliothèque de philosophie contemporaine).

Possekel, U. 1999 : Evidence of Greek Philosophical Concepts in the Writings of Ephrem the Syrian, Paris / Louvain, 1999 (Corpus Scriptorum Christianorum Orientalium. Subsidia, 102).

- 2007 : «Bardaisan of Edessa : Philosopher or Theologian ? », Zeitschrift für Antikes Christentum / Journal of Ancient Christianity, 10/3 (2007), p. 442-461.

ReIs, B. 1999 (éd.) : Albinus, Der Platoniker Albinos und sein sogennanter Prologos : Prolegomena, Überlieferungsgeschichte, kritische Edition und Übersetzung, Wiesbaden, 1999 (Serta Graeca, 7).

Sambursky, S. 1959 : Physics of the Stoics, London, 1959.

- 1977 : «Place and Space in Late Neoplatonism », Studies in History and Philosophy of Science (Part A), 8/3 (1977), p. 173-187.

Savinel, P. 1962 (trad.) : Philon d'Alexandrie, Les ceuvres de Philon d'Alexandrie, 19. De Somniis I-II, Paris, 1962.

Sedley, D. 1982 : « The Stoic Criterion of Identity », Phronesis, 27/3 (1982), p. $255-275$.

Sinko, T. 1905 : De Apulei et Albini doctrinae Platonicae adumbratione, Krakow, 1905 (Rozprawy Akademii Umiejętności. Wydział Filologiczny, 2, 26).

SorabjI, R. 1988 : Matter, Space and Motion : Theories in Antiquity and Their Sequel, London, 1988.

Spanneut, M. 1957 : Lestoïcisme des Pères del'Église: de Clément de Rome à Clément d'Alexandrie, nouv. éd. rev. et augmentée, Paris, 1957 (Patristica Sorbonensia, 1).

TEIXIDOR, J. 1992 : Bardesane d'Edesse : la première philosophie syriaque, Paris, 1992 (Patrimoines. Christianisme).

- 1994: «Bardesane de Syrie », dans R. Goulet (dir), Dictionnaire des philosophes antiques, vol. 2 : Babélyca d'Argos à Dyscolius, Paris, 1994, p. 54-63.

ToDD, R. B. 1977 : « The author of the De qualitatibus incorporeis, if not Albinus, who ? », L'Antiquité classique, 46 (1977), p. 198-204. 
Whittaker, J. 1974 : « Parisinus Graecus 1962 and the Writings of Albinus », part. 1 and 2, Phoenix, 28/3 (1974), p. 320-354 et 28/4 (1974), p. 450-456.

- $1989 \mathrm{a}$ : «Albinos », dans R. Goulet (dir.), Dictionnaire des philosophes antiques, vol. 1 : Abam (m)on à Axiothéa, Paris, 1989, p. 96-97.

- $1989 \mathrm{~b}$ : « The value of indirect tradition in the establishment of Greek philosophical texts or the art of misquotations », dans J. N. Grant (éd.), Editing Greek and Latin Texts: Papers given at the twenty-third annual conference on editorial problems, University of Toronto, 6-7 November 1987, New York, 1989, p. 63-95.

- 1990 (éd.) : Alcinoos, Enseignement des doctrines de Platon, introd., texte établi et commenté, trad. par Pierre Louis, Paris, 1990 (Collection des universités de France. Série grecque, 336).

Witt, R. E. 1937 : Albinus and the History of Middle Platonism, Cambridge, 1937 (Cambridge Classical Studies).

Zagdoun, M.-A. 2000 : La Philosophie stö̈cienne de l'art, Paris, 2000 (CNRS Philosophie). 